\title{
Holographic Barcode Scanners: Applications, Performance, and Design
}

\section{LeRoy D. Dickson}

Wasatch Photonics, Inc.

Logan, Utah, USA

\section{Timothy A. Good}

Metrologic Instruments, Inc.

Blackwood, New Jersey, USA

\section{CONTENTS}

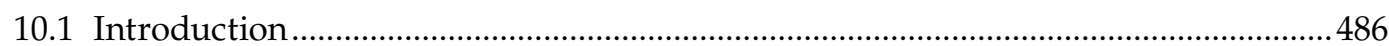

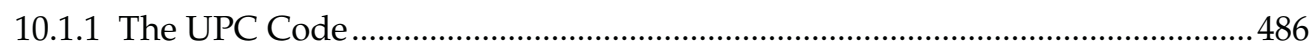

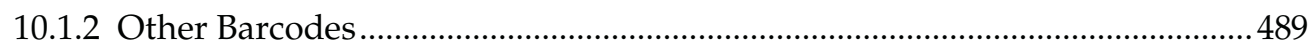

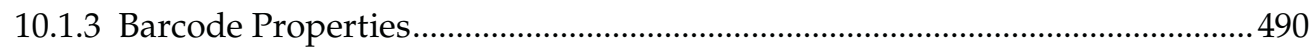

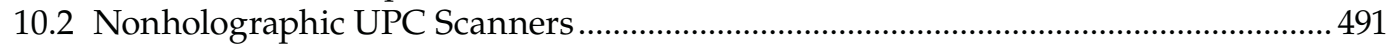

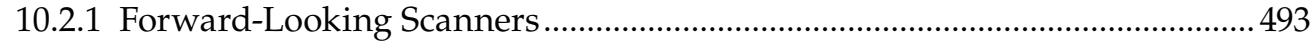

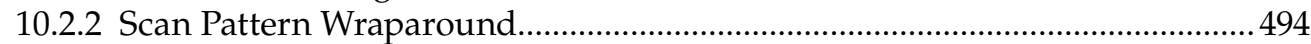

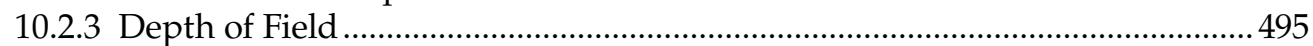

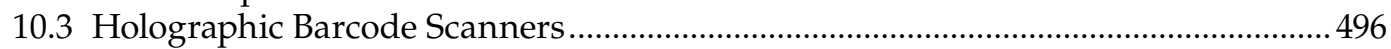

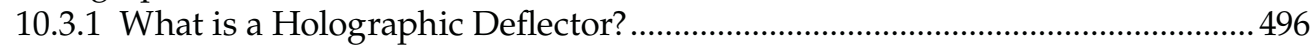

10.3.2 Novel Properties of Holographic Barcode Scanning........................................499

10.3.3 Depth of Field for a Conventional Optics Barcode Scanner .............................500

10.3.4 Depth of Field for a Holographic Barcode Scanner .........................................502

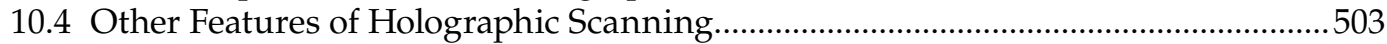

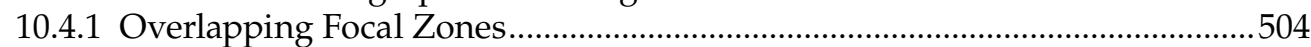

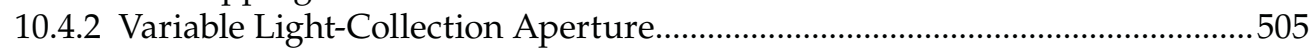

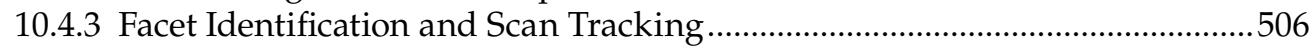

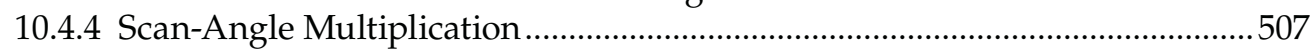

10.5 Holographic Deflector Media for Holographic Barcode Scanners ............................ 509

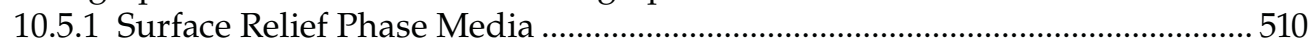

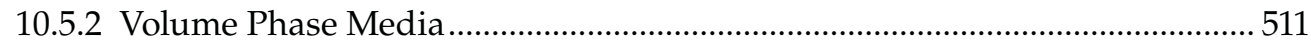

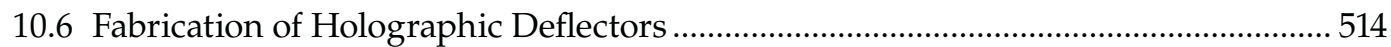

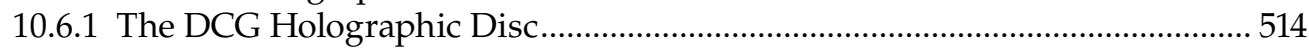

10.6.2 The Mechanically Replicated Surface-Relief Holographic Disc ..................... 517

10.7 An Example of a Holographic Barcode Scanner: The Metrologic Penta Scanner .... 518

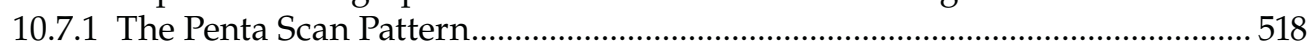

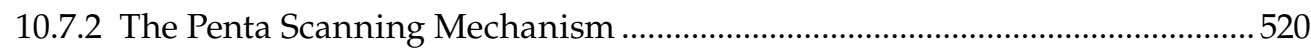

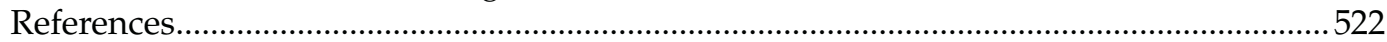




\subsection{INTRODUCTION}

Significant changes have occurred in the field of laser scanning since the first edition of this book was published over 10 years ago. Specifically, visible laser diodes (VLDs) have become the laser light source of choice in the scanning industry, allowing scanners to become much smaller, in the form of hand-held and wearable scanners. Holographic scanning, however, does not yet have a very significant presence in these applications.

In the first edition, much attention was given to supermarket scanners and most of the examples were given in reference to such designs. Over the past decade, however, a great deal of growth has occurred in the industrial scanning market, and the adaptability of holography has helped it grow into this market more significantly. Accordingly, more examples are given in this edition with respect to the industrial scanning market.

The fundamentals of scanning, however, have not changed. Neither has the presence of several decades of barcode and scanner design specifications and laser standards been diminished. For example, printing specifications of barcodes are based on reflectance properties at the wavelength of helium-neon lasers, predominantly used years ago but no longer in scanners today. As such we will begin with a basic discussion of barcodes.

A barcode is a sequence of dark bars on a light background or the equivalent of this with respect to the light reflecting properties of the surface. The coding is contained in the relative widths or spacings of the dark bars and light spaces. Perhaps the most familiar barcode is the universal product code (UPC), which appears on nearly all of the grocery items in supermarkets today. Figure 10.1 is an example of a UPC.

A barcode scanner is an optical device that reads the code by scanning a focused beam of light, generally a laser beam, across the barcode and detecting the variations in reflected light. The scanner converts these light variations into electrical variations, which are subsequently digitized and fed into the decoding unit, which is programmed to convert the relative widths of the digitized dark/light spacings into numbers and/or letters.

The concept of barcode scanning for automatic identification purposes was first proposed by N. J. Woodland and B. Silver in a patent application filed in 1949. A patent, titled "Classifying Apparatus and Method," was granted in 1952 as U.S. Patent No. 2,612,994. This patent contained many of the concepts that would later appear in barcode scanning systems designed to read the UPC.

\subsubsection{The UPC Code}

In the early 1970s, the supermarket industry recognized a need for greater efficiency and productivity in their stores. Representatives of the various grocery manufacturers and

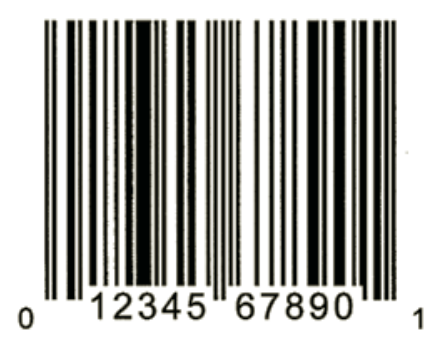

FIGURE 10.1

Typical UPC barcode. 
supermarket chains formed a committee to investigate the possibility of applying a coded symbol to all grocery items to allow automatic identification of the product at the checkout counter. This committee, the Uniform Grocery Product Code Council, Inc., established a symbol standardization subcommittee, whose purpose was to solicit and review suggestions from vendors for a standard product code to be applied to all supermarket items.

On April 3, 1973, the Uniform Grocery Product Code Council announced their choice. The code chosen was a linear barcode similar to a design proposed by IBM. The characteristics of this barcode, the now familiar UPC, are described in detail in the article by Savir and Laurer. ${ }^{1}$

The UPC is a fixed-length numeric-only code. It consists of a pair of left guard bars, a pair of right guard bars, and, in the standard version A symbol shown in Figure 10.1, a pair of center guard bars. Each character is represented by two dark bars and two light spaces. The version A symbol contains 12 characters, six in the left half and six in the right half. Thus, a version A UPC symbol will have 30 dark bars and 29 light spaces, counting the six guard bars-left, right, and center. The first character in the left half is always a number system character. For example, grocery items are given the number system 0 , which often appears on the left of the symbol. The last character on the right is always a check character. This sometimes appears to the right of the barcode symbol.

The remaining five characters in the left half of the version A UPC symbol identify the manufacturer of the product. For example, the left-half number 20000 represents Green Giant products. This left-half five-digit code is assigned to the various manufacturers by the Uniform Product Code Council.

The remaining five characters in the right half of the version A UPC symbol identify the particular product. This right-half five-digit code is assigned by the product manufacturer at his discretion. For example, Green Giant has assigned the right-half number 10473 to their $17 \mathrm{oz}$. can of corn. Therefore, the complete UPC code for the Green Giant $17 \mathrm{oz}$. can of corn, ignoring the number system character and the check character, is 20000-10473.

There are a number of other properties of the UPC code and symbol that are significant relative to the design and use of equipment for reading the code. First, the left and right halves of the version A symbol are independent. That is, each half can be read independently of the other half and then combined with the other half in the logic portion of the reader to yield the full UPC code. Furthermore, as shown in Figure 10.2, each half of the UPC symbol is "over-square." That is, the symbol dimension parallel to the bars is greater than the symbol dimension perpendicular to the bars. The aspect ratio of the barcode is vital in the determination of a minimum scan pattern for reading the code, as we will see in a later discussion.
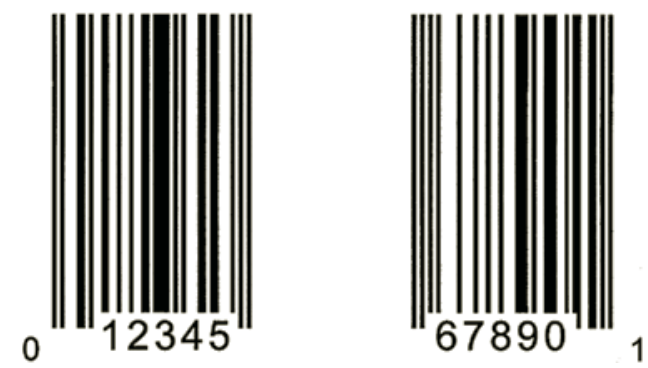

FIGURE 10.2

Two UPC half-symbols. 
It should be noted that the original "over-square" design of the UPC code is not always adhered to by product manufacturers. Often they will truncate the height of the code. These truncated codes, while discouraged by the Uniform Product Code Council, are often used by manufacturers to maximize the space available for product information. That, in turn, has meant that scanner designs must be more complex and the decoding algorithms more sophisticated.

Each character of the code is represented by two dark bars and two light spaces. Individual bars and spaces can vary in width from one module wide to four modules wide. (Note, on all UPC codes, the guard bars are always one module wide and separated by a one-module-wide space.) The total number of modules in each character is always seven. The left-half characters are coded inversely from the right-half characters. As shown in Figure 10.3, for example, if we let white $=0$, and black $=1$, then the code for the number 2 in the left half is 0010011 while in the right half it is 1101100.

The fact that each character is always seven modules wide leads to a second major property of the UPC code: it is self-clocking. Therefore, absolute time measurements are unimportant. What is measured is the time that is required to go from the leading edge of the first black bar to the leading edge of the third black bar (i.e., the first black bar of the next character). This time interval is then divided into seven equal intervals, and the relative widths of the two black bars and the two white spaces are then determined for decoding purposes. Thus, the total width of a character-black-white-black-white-is measured, and the relative widths of the two black bars and the two white spaces are determined for decoding.

This self-clocking feature is very important in the design of scanners for reading the UPC code. It means that the velocity of a scanning light beam for reading the code does not have to be constant across the full width of the code. The velocity only needs to be reasonably constant across a single character. This means that the UPC code can be read by moderately nonlinear scan patterns, such as sinusoidal or Lissajous patterns. It also means that the code can be read on curved surfaces. Furthermore, the scan lines reading the code do not have to be perpendicular to the bars and spaces in the code. As originally designed, satisfactory reading of a UPC code can be obtained with scan lines that pass through the code at any angle relative to the bars and spaces so long as a single scan line passes completely through a full half symbol, including the center guard bars and one pair of edge guard bars. More sophisticated decoding algorithms now exist that can "stitch" together three smaller, individually scanned pieces of a UPC code (or other symbol), and, as such, the process is commonly referred to as stitching. The use of stitching allows a slightly less thorough scan pattern to do as good a job as a better pattern and makes a good pattern even better; however, the UPC code was designed with only the left and right sides in mind. Stitching was a software adaptation developed later on. ${ }^{2}$ One of the factors driving its development was the occurrence of the aforementioned truncated codes, for which stitching is particularly useful.

(a)

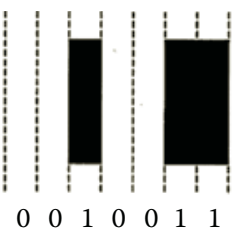

(b)

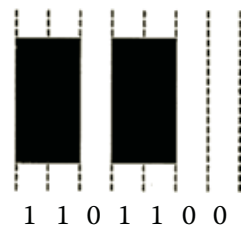

FIGURE 10.3

An example of character encoding for the number 2: (a) left-half character; (b) right-half character. 
A third property of the UPC symbol of significance to the scanner designer is the size of the symbol, which is allowed to vary from the nominal size (about 1.0 in $\times 1.25$ in [25.4 mm $\times 31.75 \mathrm{~mm}$ ] for the version A symbol) down to $0.8 \times$ nominal and up to $2.0 \times$ nominal. This size variation allows the use of small labels on small packages with good print quality and large labels on larger packages with poorer print quality. From the scanner designer's viewpoint, the small label will establish the minimum bar width to be read and the large label will set a lower limit on the size of the scan pattern.

The minimum bar width established by the UPC specification, including tolerances, is 0.008 in $(0.2 \mathrm{~mm})$. This number establishes the maximum attainable depth of field for the optical reader. In practice, the depth of field of the typical laser scanner designed to read the UPC code will easily meet, and exceed, the $1 \mathrm{in}(25.4 \mathrm{~mm})$ depth of field required by the early UPC guidelines. However, this 1 in depth of field did not take into consideration the manner in which the scanners would eventually be used. Depths of field of several inches $(100 \mathrm{~mm}+)$ are required for today's UPC barcode readers.

Finally, the contrast specification for the UPC symbol requires that the contrast be measured using a photomultiplier detector (PMT) with an S-4 photocathode response curve coupled with a Wratten 26 filter. This combination has a peak response at a wavelength of approximately $610 \mathrm{~nm}(24 \mu \mathrm{in})$, falling to zero at approximately $590 \mathrm{~nm}(23.2 \mu \mathrm{in})$ and $650 \mathrm{~nm}(25.6 \mu \mathrm{in})$. This response includes, not coincidentally, the wavelength of the helium-neon laser, $632.8 \mathrm{~nm}(24.9 \mu \mathrm{in})$, which was the preferred laser at the time that laser scanners were first being considered. While several of the inks used for printing UPC labels can provide acceptable contrast out to $700 \mathrm{~nm}(27.56 \mu \mathrm{in})$ there are many other inks in use that do not provide acceptable contrast beyond $650 \mathrm{~nm}$ (25.6 $\mu \mathrm{in})$. These inks would preclude general use of longer-wavelength light sources. Today, nearly all UPC scanners use one or more diode lasers as their light source(s); however, the wavelengths of these lasers fall within the original UPC wavelength specification.

\subsubsection{Other Barcodes}

The UPC code is not widely used in the industrial environment (the manufacturing, warehouse, and distribution applications). Here, the requirements are different from those of the supermarket, so the codes used are different than the UPC code. The preferred codes for the industrial environment are Bar Code 39, Interleaved 2 of 5, and Codabar.

The most common barcode in the industrial environment is the so-called Bar Code 39, or 3 of 9 barcode. This code is fully alphanumeric and is self-checking. For a full discussion of Bar Code 39, as well as several other codes, and the definition of such terms as "self-checking," see work by Allais. ${ }^{3}$ The code shown in Figure 10.4 is an example of Bar Code 39.

Bar Code 39 got its name from the fact that it originally encoded 39 characters: the 26 letters of the alphabet, the numbers from 0 through 9 , and the symbols -.., and SPACE, plus a unique start/stop character, the asterisk $\left(^{*}\right)$. Today it also encodes the four so-called special

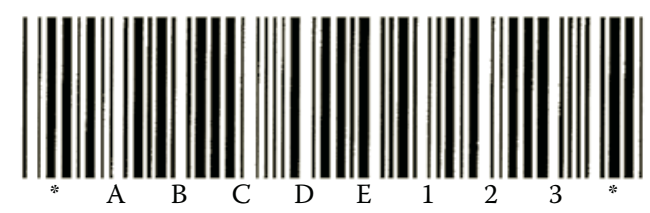

FIGURE 10.4

An example of Bar Code 39. 
characters: $\$, /,+$, and $\%$, for a total of 43 characters. However, it is still referred to as Bar Code 39. It is also often called the 3 of 9 code, because each character in the code is represented by nine elements (five dark bars and four light spaces), and three of them are wide with the remaining six narrow. In the primary set of 39 characters, two of the wide elements are dark bars. In the four special characters, the wide elements are all light spaces.

The 2 of 5 code is a subset of the 3 of 9 code. In 2 of 5 , only the bars are used for encodation. Two of the five bars are wide, just as in the original 3 of 9 code. The spaces are not used. This code is strictly numeric. The basic 2 of 5 code is not widely used in the industrial environment, but a variation of it, called the Interleaved 2 of 5 code, is used extensively for manufacturing and distribution applications. This code uses the bars to encode one character in the standard 2 of 5 code and then uses the interleaving spaces to encode a second character in 2 of 5 code. This allows more characters to be encoded in a fixed barcode length than either 3 of 9 code or 2 of 5 code. This code is also only numeric, but, due to the interleaving feature, it can encode nearly $80 \%$ more characters per unit length than Bar Code 39, assuming both codes have the same minimum bar width. For this reason, the Interleaved 2 of 5 code is often used where space limitations will not permit the use of Bar Code 39.

A third code that is used extensively in medical institutions, and which was adopted as an early standard by the American Blood Commission for use in identifying blood bags, is Codabar. This code is also frequently seen in some transportation and distribution applications.

\subsubsection{Barcode Properties}

From the standpoint of the scanner, the important properties of any barcode are:

1. Minimum bar width: generally specified in millimeters or mils (thousandths of an inch). And often referred to as the " $X$ " dimension.

2. Contrast: a measure of the reflectance of the bars and spaces. Contrast is generally expressed in terms of the print contrast signal (PCS), defined as

$$
\mathrm{PCS}=\frac{r_{\mathrm{s}}-r_{\mathrm{b}}}{r_{\mathrm{s}}}
$$

where $r_{\mathrm{s}}$ is the reflectance of a space and $r_{\mathrm{b}}$ is the reflectance of a bar. It should be noted that PCS is usually measured for one particular wavelength of light. In the majority of applications this wavelength is $633 \mathrm{~nm}$ (24.9 $\mu \mathrm{in})$, the wavelength of the helium-neon laser, which was the most common light source for most of the early laser scanners. (Some applications allow PCS to be measured at $900 \mathrm{~nm}$ [35.4 $\mu \mathrm{in}]$, the wavelength of some infrared light sources used in some readers.) This is an important point to remember since PCS will vary drastically as a function of wavelength if colored inks or backgrounds are used. In practice, the most important reflectance property of the barcode is the absolute contrast, which is simply the space reflectance minus the bar reflectance (the numerator in Equation 10.1).

3. Code length: the physical length of a barcode is determined by the density of the code (which is determined by the minimum bar width) and the number of characters in the code. The physical length of the code determines how long the scan lines must be and, when combined with the code height, will determine how accurately the scan line must be oriented with respect to the barcode. 
4. Code height: the height of the barcode (the dimension parallel to the bars) will determine the angular accuracy required in orienting the scan line relative to the barcode.

5. Barcode quality: this includes both the quality of the printing or etching of the code itself and the quality of the surface on which the code is printed. Obviously, the better the quality of both, the easier it will be for the scanner to successfully scan and decode the barcode.

There is a great deal more that could be said about the barcodes themselves. However, a more detailed analysis of the fundamental properties of the barcodes is beyond the scope of this review and is not really necessary for the purposes of our discussion of barcode scanning.

\subsection{NONHOLOGRAPHIC UPC SCANNERS}

A block diagram of a typical laser scanner system for reading the UPC code is shown in Figure 10.5. The focused laser beam scans the UPC symbol on a package as the package passes over the read window of the scanner. The laser beam is reflected from the symbol as it passes over the dark bars and light spaces. The diffuse portion of the reflected light is modulated by the reflectivity variations of the symbol (bars and spaces). This light modulation is detected by the photodetector, which converts the light modulation into electrical modulation. The electrical "signal" is then amplified, digitized, and transmitted to the "candidate select" block. This block acts as a filter, allowing only valid UPC halfsymbols to pass to the decoder. The decoder converts the signals for each half-symbol into characters and then combines the characters for the two half-symbols together to yield a complete UPC product identification code. The computer then searches its memory for a description and price of the item identified by this UPC code. This information is transmitted back to the checkout terminal where it appears on the display and the customer receipt. Simultaneously, the store inventory is updated to reflect the sale of the identified item. All of this takes place in a few milliseconds.

The focused spot size of the scanning laser beam must be about $0.2 \mathrm{~mm}$ in order to be able to read the labels with the smallest bar widths while still yielding adequate depth of

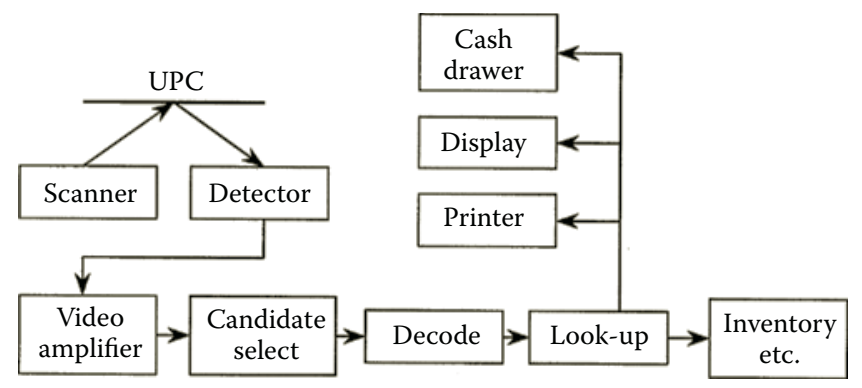

FIGURE 10.5

Block diagram of a UPC scanner. 
field. This requires an optical $f$-number of approximately 250 , which, when combined with scanner geometry, establishes the focusing optics' requirements.

A number of technologies are available for deflecting the focused laser beam in a conventional, nonholographic, UPC scanner. Cost and performance requirements limit the choice to mechanical deflectors-generally either rotating or oscillating mirrors, or a combination of these. The scan pattern created by the laser deflection mechanism must be capable of reading a full-size version A UPC symbol regardless of the orientation of the symbol in the scan window. In other words, the scanner must be omnidirectional. An omnidirectional scanner will allow maximum freedom for the scanner operator when bringing the item across the scan window.

We have already seen that the two halves of the UPC symbol can be read independently of each other. We have also seen that each half-symbol is over-square. Therefore, the minimum scan pattern that will allow omnidirectional scanning is a pair of perpendicular scan lines in the form of an X (see Figure 10.6a). As the UPC symbol passes over the scan window, at least one of the legs of the $X$ will pass through the entire half-symbol at some point in the window. Figure 10.6a shows two extreme orientations of the symbol as it is passed over the window. These are the worst-case orientations in that they allow the minimum time for scanning the symbol satisfactorily.

The amount that the half-symbol is over-square, when combined with the maximum item velocity of $2.54 \mathrm{~m} / \mathrm{s}$ (100 in/s), determines the minimum pattern repetition rate to guarantee at least one good scan through the symbol as it passes across the scan window, regardless of its orientation. The pattern repetition rate, the total scan length, and the width of the smallest UPC module establishes the maximum video signal rate seen by the photodetector.

Although the pattern in Figure 10.6a is the minimum scan pattern required to yield an omnidirectional scanner for the UPC symbol, it is not an "optimum" pattern. The pattern repetition rate required to guarantee one scan through a UPC half-symbol moving across this pattern at $2.54 \mathrm{~m} / \mathrm{s}$ (100 in/s), at the worst-case orientation relative to the scan pattern, is very high. This results in high scanning spot velocities and subsequently high video signal rates. A "better" scan pattern can guarantee one good scan at lower pattern repetition rates and lower scan velocities. An optimum scan pattern will minimize scan velocity, thereby minimizing video signal rates.

If one could increase the amount that a symbol is over-square (i.e., improve the aspect ratio), then one could reduce the pattern repetition rate, and the scan velocity, and still guarantee one good scan through the UPC half-symbol at maximum symbol velocity and worst-case symbol orientation. While the symbol itself cannot be changed, one can effectively improve the aspect ratio of the symbol by using a scan pattern where the scan lines are separated by angles less than $90^{\circ}$. Thus, a scan pattern consisting of three scan lines,

(a)

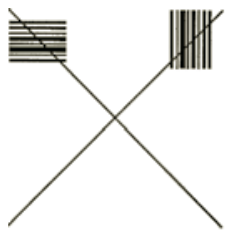

(b)

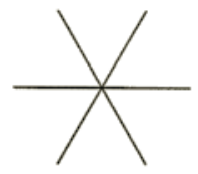

(c)

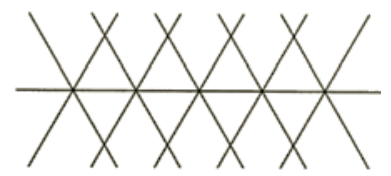

FIGURE 10.6

Omnidirectional scan patterns in the plane of the window: (a) the minimum scan pattern; (b) basic pattern of an optimum scan pattern; (c) optimum pattern for a rectangular window. 
for example, instead of two, could be repeated less often and still be able to read the UPC symbol under the worst-case conditions mentioned above. Increasing the number of scan lines has the effect of increasing the total linear distance scanned by the scan pattern, which, by itself, would increase the scan velocity of the scanning spot. However, the reduction in the pattern repetition rate is greater than the increase in the scan length. The net result is a better scan pattern, in the sense described above.

Can we continue to improve the scan pattern by adding still more lines? Unfortunately, the answer is no. The reduction in the required pattern repetition rate realized by using four scan lines is nearly offset by the increased scan velocity resulting from the greater distance traveled by the scanning spot. The small amount of gain is not enough to justify the increased cost and complexity required to generate the four-line pattern. Beyond four lines, there is no gain when scanning UPC symbols. (We will, however, see later that in certain industrial applications four- and five-line patterns can be quite effective, especially when scanning symbologies with extreme aspect ratios.) It appears, then, that for the UPC code the optimum scan pattern in the plane of the window would be one based on the three-line pattern shown in Figure 10.6b. This fundamental three-line pattern, which is still the basic criterion used in the design of UPC scanners in 2004, formed the basis of the first scanner designed to read the UPC code, the IBM 3666 scanner. The linear equivalent of the Lissajous scan pattern used in the IBM 3666 scanner is shown in Figure 10.6c.

\subsubsection{Forward-Looking Scanners}

Initially, all UPC scanners were conceived as "bottom scanners." That is, the scanning laser beam pointed directly upward to read the UPC symbols on the bottoms of packages as they passed over the scan window. A major problem was encountered in the design of this type of scanner. UPC symbols printed on shiny surfaces were difficult to read because the specular reflection from the shiny surfaces contained no bar-space modulation in the specularly reflected light. In addition, the specular reflection created saturation problems in the photodetector because the specularly reflected light was so much more intense than the diffusely reflected light. In most scanners, the photodetector was located back along the general direction of the outgoing laser beam. Therefore, some solution had to be found that would keep the specularly reflected light from being directed back along the laser beam path.

One solution to this problem is shown in Figure 10.7a. The laser beam is tilted at an angle of approximately $45^{\circ}$ relative to the scanner window. In this configuration, the specularly reflected light is reflected away from the photodetector, thereby eliminating the specular reflection problem.

(a)

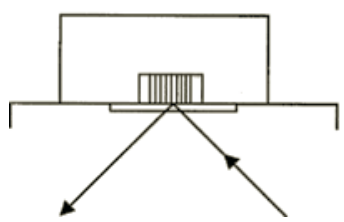

(b)

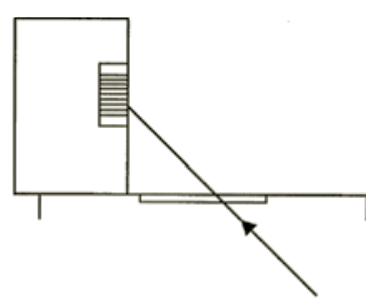

FIGURE 10.7

Tilted-beam scanning: (a) removing the specular reflection problem; (b) side reading with a forward-looking tilted beam. 
A fringe benefit occurs when this scanner geometry is used. The tilted beam can be used to read UPC symbols on the front of packages without tilting the packages forward (see Figure 10.7b). Of course, this increases the depth of field required to read these upright labels, but the laser scanner has the capability to provide a depth of field of several inches $(100 \mathrm{~mm}+)$, which is usually sufficient for side reading with the tilted beam geometry. Nearly all UPC scanners today employ some form of forward-looking, tilted-beam reading geometry.

\subsubsection{Scan Pattern Wraparound}

The next development in the evolution of the scan pattern was the introduction of scan pattern wraparound. Several scan patterns were introduced that took the basic three-line optimum scan element shown above and created it in such a way that the scan lines were directed at the items from points within the scanner that were slightly off to the sides of the package. In these scanners, horizontal lines were projected forward from immediately in front of the item and from directions slightly off to each side. Vertical lines were also projected from slightly off to each side. The pattern projected from the two sides was essentially a cross pattern, while the scan pattern projected from immediately in front of the item was a horizontal line, as shown in Figure 10.8. The overall pattern was created by using a rotating mirror deflector and an array of fixed folding mirrors.

This type of scan pattern was effective in reading the UPC symbol on the scan window because it employed the basic three-line optimum scan element. It was also effective in reading upright items because it projected a pattern of perpendicular horizontal and vertical lines on the front of the items. Such a pattern is effective for upright reading because the bars in the UPC symbol will usually be either vertical or horizontal when the package is presented to the scanner in this manner.

The major advantage of this type of scan pattern is that it "wraps around" to the sides of the packages to some degree. This means that the operator does not have to align the item as carefully when he brings the item across the scan window. The UPC symbol can be on the bottom of the package, on the front of the package, or on the side of the package and still be readable by the scanner. This has a positive effect on operator productivity.

This concept of wraparound was further exploited in the development of "bi-optic" scanners, exemplified by the NCR 7875, the PSC Magellan SL, and the Metrologie Stratos.

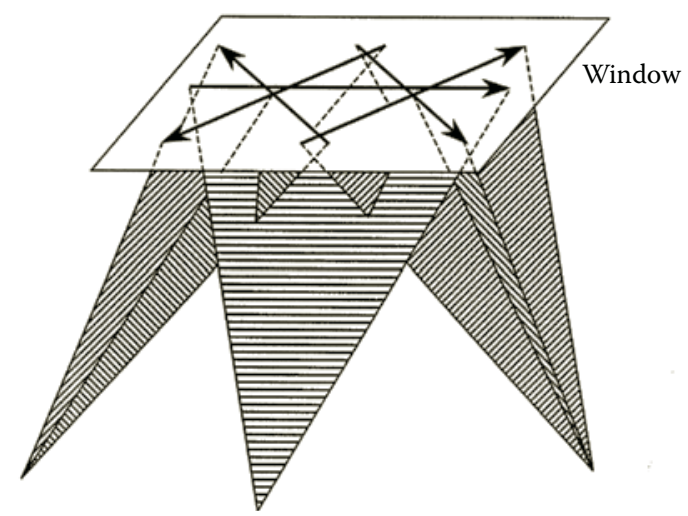

FIGURE 10.8

Projected wraparound scan pattern. 
The bi-optic-type scanner is currently becoming the most used type of scanner in supermarkets and other large-volume, point-of-sale applications. The bi-optic nature of the scanner refers to the two separate scan windows it possesses, as shown in Figure 10.9. In this configuration there is a horizontal scan window and a second window at or near vertical orientation, depending on the specific scanner in question.

Improved performance is gained by employing the wraparound concept from both windows. In the preferred package-presentation orientation, two of the six faces of an item directly face a scanner window and are seen by the primary three-line pattern. Two other faces, typically the faces in the direction of item motion (see Figure 10.9), are targeted by the wraparound patterns of both scanner windows. The package surface that faces away from the vertical window still potentially sees the wraparound pattern out of the horizontal window. Finally the top surface of the package, which has the least exposure to the scan pattern, may still have some chance of being scanned by the wraparound pattern of the vertical window, depending on the package height and code orientation. The net result is effective scanning throughout the majority of the $360^{\circ}$ horizontal orientation range and equally effective scanning through nearly $270^{\circ}$ of vertical orientation. Such a wide range of acceptable presentation orientations means very little of an operator's time needs to be spent paying attention to the position and orientation of a code on an item.

\subsubsection{Depth of Field}

The multidirectional, three-line scan pattern forms the basis for nearly all present-day UPC scanners, holographic and nonholographic. Unfortunately, the forward-looking feature increases the depth of field requirement considerably. As much as 150-mm (6-in) depth of field may be required to read some barcodes on upright items. Because the codes must, in many cases, be read by a tilted beam, the resultant spot ellipticity on the barcode will increase the effective scanning spot diameter. This will reduce the depth of field of the scanner.

Providing satisfactory scanning performance over such a large depth of field, with a tilted scanning beam, is a significant challenge to the scanner designer. Significant improvements in signal processing over the last decade have allowed smaller bar widths to be read without reducing the actual size of the spot, helping to solve this problem and increase depth of field. Another means of easing the problem, without relying on electronic improvements, is to design a scanner that can provide more than one focal plane. Such a scanner could focus

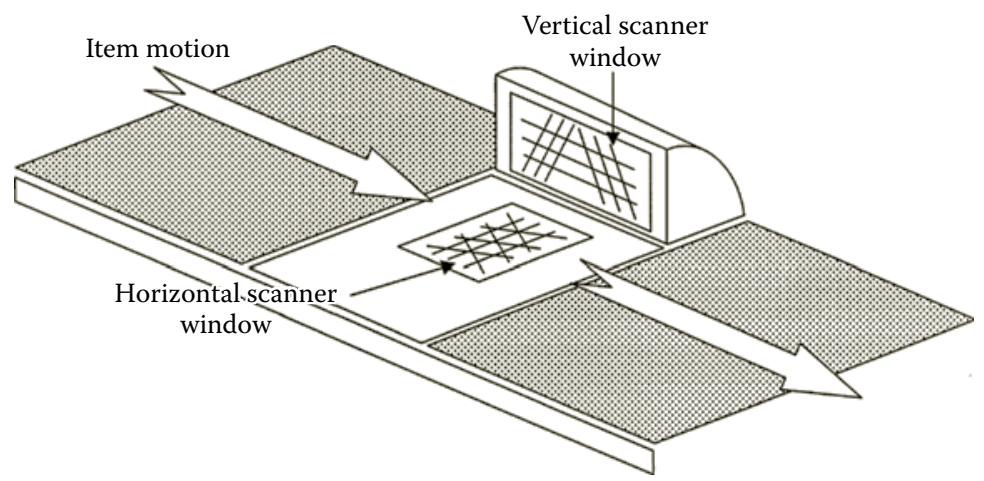

FIGURE 10.9

A "bi-optic" supermarket scanner. 
some of its scan lines close to the scan window and some of its lines further from the scan window, thereby increasing the effective depth of field of the scanner.

Holographic scanning allows the scanner designer to add this additional degree of flexibility to the scanner. The holographic scanning element essentially allows each scan line to be optimally focused to provide increased depth of field and increased flexibility in the placement of beam-folding mirrors for the creation of the scan pattern. This also allows for a more complex, and more effective, scan pattern. The need for greater depth of field and the desire for a more effective scan pattern led to the development of the holographic barcode scanner.

\subsection{HOLOGRAPHIC BARCODE SCANNERS}

The concept of holographic scanning has been around for three decades, ${ }^{4}$ and during this time many different applications have been suggested, 5,6 but few have been demonstrated and an even smaller number have made it into the marketplace. A general review of holographic scanning and various applications can be found in the book by Beiser ${ }^{7}$ as well as in previous volumes of optical engineering. ${ }^{8}$

Holographic barcode scanners first appeared commercially in 1980 with the introduction of holographic UPC scanners by IBM and Fujitsu. Today, Metrologie manufactures holographic scanners primarily for industrial applications. Such applications range from large depth of field (greater than $1 \mathrm{~m}$ [40 in]) overhead scanners, to high-density, highresolution scanners for large aspect-ratio codes, to completely automated hands-off scanning tunnels for bulk-mail centers.

\subsubsection{What is a Holographic Deflector?}

Photography is a light-recording process in which a two-dimensional light-intensity distribution incident on a light-sensitive medium is recorded by that medium. In contrast, holography is a light-recording process in which both the amplitude and phase distribution of a complex wavefront incident on the recording medium can be recorded by that medium.

Holography, therefore, differs from photography in that it is able to record all of the information that is needed by the eye, or any other optical system, to interpret the full three-dimensional nature of the object.,10 This information is accessed when the recording (the "hologram") is illuminated by the proper light source-usually, but not always, a laser.

The most common form of hologram creates, when viewed, a three-dimensional image of a complex, three-dimensional object. The reduction of the three-dimensional object to a single point-source produces a special case of particular importance to deflection-a hologram that acts as a lens to focus an incident laser beam. This type of hologram is referred to as a holographic optical element (HOE).

The concept of holographic recording and reconstruction-more importantly, how it deflects light—can best be understood with reference to Figures 10.10 and 10.11. In Figure $10.10 \mathrm{a}$, two wavefronts of equal intensity created from a laser are directed to overlap in some region of space where the recording is to be made. If the optical path difference from the point of beam separation to the region of overlap is within the coherence length of the 
(a)

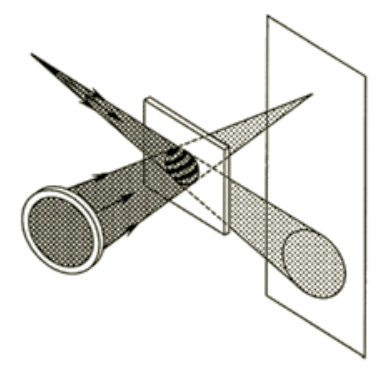

(b)

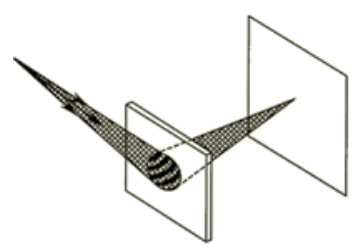

(c)

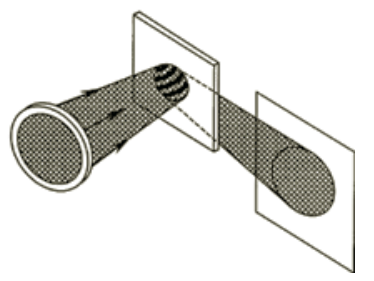

FIGURE 10.10

Simple holography: (a) recording the hologram; (b) reconstruction of the convergent wavefront (positive lens); (c) reconstruction of the divergent wavefront (negative lens).

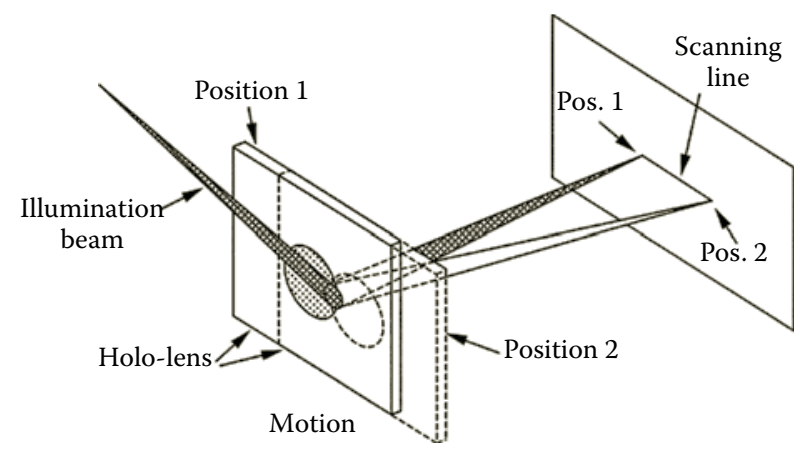

FIGURE 10.11

Principle of holographic deflection.

source, the resulting interference pattern will be stationary in both space and time and will have high fringe contrast. The intensity distribution in these fringes can be exposed onto, or more properly into, a suitable photosensitive medium such as a photographic emulsion. After processing, the recording contains a variation in optical density, refractive index, or optical thickness - sometimes a combination of all three-and is the hologram. When this recording is repositioned and illuminated by one of the wavefronts, such as the diverging wavefront in Figure 10.10b, the structure at each point within the hologram diffracts the illuminating light and creates a new wavefront that is identical to the original second wavefront. In the case of Figure $10.10 \mathrm{~b}$ the result is a converging and deflected wavefront. This simple HOE is the equivalent of a positive or converging lens in combination with a prism converting and deflecting light from a point object to a point image. The efficiency and quality with which this wavefront conversion occurs are directly related to the recording configuration and selection of recording material. In Figure 10.10c we see that 
the equivalent of a negative lens is realized by illuminating the same HOE with the converging wavefront, thereby reproducing the original diverging wavefront. Holographic recording of complex multidimensional objects can be treated as the recording of a superposition of individual spherical waves from all the points in the object field.

By using a combination of small area illumination and HOE translation, the reconstruction geometry of Figure 10.10 can be used to create simultaneous light deflection and focusing. This is shown in Figure 10.11, where the HOE is initially located at position 1 and has a small subarea, on the right side, illuminated by a diverging wavefront that corresponds to the diverging reference-construction-beam of Figure 10.10a. The light is focused by this subarea of the HOE to the point in the image plane labeled "position 1" (Figure 10.11). This point in the image plane corresponds to the location, with respect to the displaced HOE, of the original convergence point of the converging construction wavefront in Figure 10.10a. As the HOE is translated, different subareas of the HOE are passed under the illuminating beam, and the reconstructed image point is caused to translate by the same distance, in this case to position 2. This is completely analogous to the deflection and focusing that would occur if a conventional lens were illuminated off axis with a collimated beam and the lens displaced normal to its optic axis. A continuous back and forth motion in either case produces the same motion in the focused spot.

In practice, however, a continuous rotary motion rather than a reciprocating motion is easier to implement. Higher scan speeds can be realized, and different holograms can be easily accessed. Consequently, most holographic deflectors consist of a number of unique HOEs placed circumferentially as sectors on a glass disc, as shown in Figure 10.12. Other materials can be used, and other geometries besides the disc geometry can be used, ${ }^{8}$ but, for simplicity, we will restrict our discussion to the glass disc, which is the most common medium and geometry used in holographic scanners today. It should be noted that plane linear gratings, producing prismatic deviation without focal power, must be rotated to generate scanning. Translation of a plane grating in one direction will not produce scanning.

A holographic deflector disc, when properly illuminated by a laser and rotated about its axis of symmetry, can produce a complex variety of scanning laser beams. The optical and geometrical properties of each of these beams can be distinctly different from all of the others. This is the most important feature of holographic scanning. It is the major feature

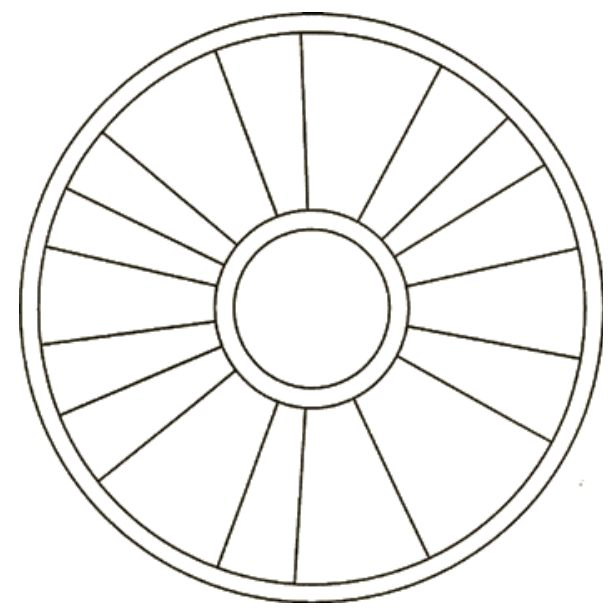

FIGURE 10.12

A holographic deflector disc. 
that distinguishes it from conventional laser scanning technology and, in a barcode scanner, allows the introduction of capabilities that could not be readily achieved with conventional technology. ${ }^{11}$

The holographic disc works in the following manner. Each sector, or facet, of the holographic disc is a unique HOE of the type previously described-the holographic equivalent of a prism and lens combined. When a facet is illuminated by a laser beam, the beam is diffracted, or bent, by the facet and focused to some point in space (see Figure 10.13). The focal length and deflection angle are established during the holographic construction of the facet and may vary from facet to facet.

As the disc rotates, the deflected, focused laser beam scans. When the beam scans across a barcode, some of the diffusely reflected light will return to the facet that generated the scanning beam. The facet now acts as a light-collection lens, combined with a prism, to collect a portion of the reflected light and direct it toward a photodetector. ${ }^{12}$

\subsubsection{Novel Properties of Holographic Barcode Scanning}

The use of holography in barcode scanning allows the introduction of scanning concepts that are not available to the designer of conventional barcode scanners, at least not in any economically practical design. Such concepts as multiple focal planes, overlapping focal zones, variable light-collection aperture, facet identification, and scan-angle magnification allow holographic scanning to bring to barcode scanning some significant design and performance capabilities.

A conventional barcode scanner contains a lens for focusing the laser beam, a device for deflecting the laser beam, and some optics for collecting a portion of the laser light reflected from the barcode and focusing it onto the photodetector. In a holographic scanner, all of these properties-focusing, deflecting, and light collection-are contained in the holographic disc. As indicated earlier, these properties may be different in each sector of the holographic disc; thus, a 16-sector holographic disc, for example, would contain 16 unique optical systems. Each of these systems would have its own focal length, scan angle, and light-collection aperture. One revolution of such a holographic disc would produce the equivalent of scanning with 16 different scanners.

Because each facet of the holographic disc may be different, with its own combination of focal length, deflection angle, and facet area, then one complete rotation of the disc will create multiple scan lines with multiple deflection angles, multiple focal lengths, and multiple light-collection systems. This enables the holographic scanner to introduce some novel operational characteristics.

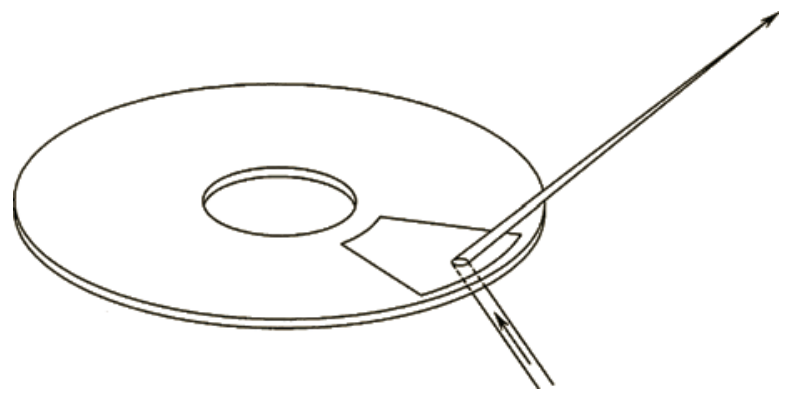

FIGURE 10.13

Light deflection and focusing by a holographic disc. 
One of the major advantages of using a holographic disc in a barcode scanner is that it can provide a much larger depth of field than would be attainable with a conventional, single-focal-length, barcode scanner. In order to understand this point, we need to briefly review the subject of depth of field.

\subsubsection{Depth of Field for a Conventional Optics Barcode Scanner}

In barcode scanning, depth of field is the distance along the laser beam, centered around the focal point of the scanner, over which the barcode can be successfully scanned. The spot size profile of a laser beam along its direction of propagation is established by the beam waist diameter and the wavelength of the laser light source. The depth of field of a barcode scanner employing such a beam depends on the size of the minimum bar width in the barcode being read and also on the resolving ability of the scanner electronics. For a given resolving ability there will be a normalized spot-size definition, or resolution criterion ( $C$ in Equations 10.3 through 10.5), based on the assumed Gaussian intensity profile of the laser beam. This resolution criterion simply defines the relative intensity level at which the beam diameter is measured. A commonly used criterion is the $1 / e^{2}$ beam width (13.5\% intensity level, $C=0.135$ ). Typical resolving abilities of scanners range from the $50 \%$ to $70 \%$ intensity levels, or possibly even higher. Once all these beam, code, and electronic parameters have been established, there is little that can be done in a conventional barcode scanner to increase the depth of field.

Figure 10.14 illustrates the concept of depth of field. The lens in the scanner focuses the laser beam to a relatively small spot size at the focal point. The diameter of the beam at the focal point is determined by the focal length of the lens, the diameter of the beam at the lens, and the wavelength of the laser being used. When the optical system of the scanner is properly designed, the minimum spot size will be somewhat smaller than the minimum bar width and will therefore be able to successfully scan the barcodes. As one moves the barcode to either side of the focal point, toward or away from the scanner, the spot size increases as the beam becomes out of focus. Eventually, a point is reached where the outof-focus spot size is larger than the minimum bar width in the barcode. When this occurs, the barcode can no longer be successfully scanned by the beam. The distance between the two points to either side of the focal point where the limit of scanning capability occurs is, by definition, the depth of field.

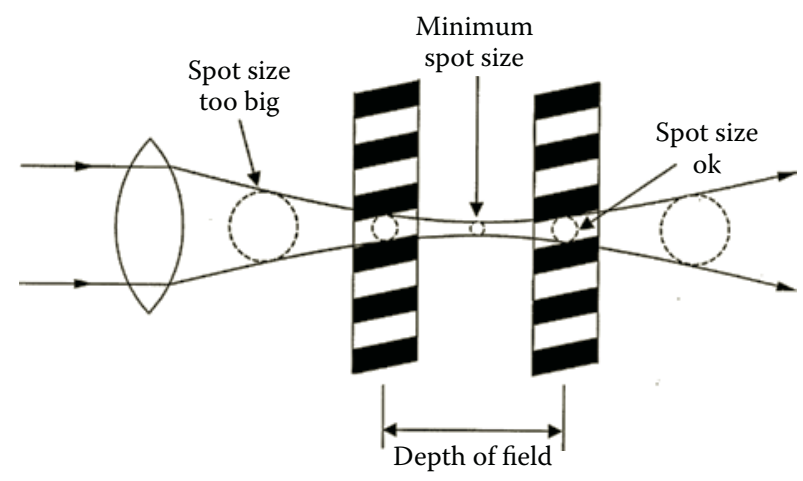

FIGURE 10.14

Depth of field for a conventional optical system. 
The major factors determining the depth of field are the spot size at the focal point, the wavelength of the laser, and the minimum bar width. (For convenience, we will assume throughout this discussion that the minimum space width is the same as the minimum bar width.) Using the notation of Dickson ${ }^{13}$ for the variation in beam radius of a propagating Gaussian beam, the $1 / e^{2}$ beam radius, $r$, at a distance, $z$, from a beam waist of $1 / e^{2}$ radius $r_{0}$ is given by

$$
r=r_{0}\left[1+\left(\frac{I z}{p r_{0}^{2}}\right)^{2}\right]^{1 / 2}
$$

The beam radius, $r_{c}$, for a different resolution criterion, $C$, is given by

$$
r_{c}=r K=r \sqrt{\frac{-\ln (C)}{2}}
$$

Similarly, the waist size at that resolution criterion is scaled down by $r_{0 c}=r_{0} K$. Equations 10.2 and 10.3 can be combined and rearranged to express the depth of field for a given beam radius, $r_{c}$, resolution criterion, $C$, and waist radius, $r_{0 c}$ as

$$
\mathrm{DOF}=\Delta z=2|z|=\frac{-4 p}{I \ln (C)} \sqrt{r_{0 c}^{2} r_{c}^{2}-r_{0 c}^{4}}
$$

It can be shown that, for any resolution criterion, the depth of field given by Equation 10.4 is maximized for a given minimum bar width when the focused spot size, as measured by the particular resolution criterion being used, is equal to the minimum bar width divided by $\sqrt{2}$. Applying that condition requires the substitutions of $2 r_{0 c}=w_{\min } / \sqrt{2}$ and $2 r_{c}=w_{\min }$ into Equation 10.4, which then reduces to

$$
\Delta z=\frac{-p w_{\min }^{2}}{21 \ln (C)}
$$

At a wavelength of $650 \mathrm{~nm}$, typical of VLDs being used in scanners today (2004), and assuming a reasonable resolution criterion of $C=0.6(60 \%)$, Equation 10.5 can be approximated as

$$
\Delta z=\frac{w_{\min }^{2}}{8.3}
$$

where $\Delta z$ is the depth of field in inches when the minimum bar width is in mils or as

$$
\Delta z=\frac{w_{\min }^{2}}{210}
$$

where $\Delta z$ is the depth of field in millimeters when the minimum bar width is in microns. 
For example, if the scanner is optimally designed to read a barcode that has a minimum bar width of 8 mils $(200 \mu \mathrm{m})$, the depth of field will be 7.7 in $(200 \mathrm{~mm})$. Note that the above equation tells us that the depth of field is strongly dependent on the size of the minimum bar width to be read. Therefore, a small minimum bar width is always accompanied by a small depth of field.

Furthermore, if the scanner is not optimally designed for the minimum bar width to be read, the depth of field will be smaller than it could be. This will be true whether the scanner is optimally designed to read either a higher density barcode or a lower density barcode. In addition, if the scanner uses a laser with a different wavelength, the depth of field will be multiplied by the ratio of the wavelength for which the design is optimized to the wavelength of the laser being used. (This assumes that the new laser has its minimum spot size focused to the same size as the original design.)

There is very little that can be done in a conventional barcode scanner to increase the depth of field. It is possible to use an autofocus scanner. Autofocus scanners have been designed and built for the industrial scanning market by Accu-Sort Systems (Telford, PA). However, a problem with such scanners is that the reaction time of the autofocus system has to be very fast to accommodate fast-moving items on a conveyor system. Because all autofocus systems today require mechanical movement of some of the optics of the scanning system, the reaction time may not be fast enough, depending on the application. In addition, such systems will add cost and complexity to the scanner.

One could also add a supplemental optical element to the scanner that could move into position in the laser beam path to change the net focal length of the scanner. This moving element would allow, for example, two different focal lengths to be selected. In practice, this approach would allow only two or three different focal lengths to be selected, giving only a slight increase in the depth of field.

Furthermore, in either an autofocus system or a dual or triple-focal-length system, only the focal lengths can be easily changed. Ideally, one should also change the aperture of the scanner as the focal length is changed. This would maintain a constant level of light collection over the full range of focus, thereby optimizing the performance of the scanner over the full range of readability. However, rapid variation of the light-collection aperture is difficult to accomplish in a conventional barcode scanner.

\subsubsection{Depth of Field for a Holographic Barcode Scanner}

The use of holography in a barcode scanner would allow the introduction of a true multifocal-plane scanner with a variable light-collection aperture. The way this would be accomplished in a holographic scanner is illustrated in Figure 10.15.

Figure 10.15 shows focusing of the laser beam by two consecutive facets on the holographic disc. Each facet will exhibit a conventional depth of field as established by the focal length of the facet, the beam diameter at the disc, and the wavelength of the laser. Notice, however, that the two facets are focused at different distances from the disc. Therefore, while each facet has only a conventional depth of field, the combined depth of field of the two facets is twice as great as for either facet alone, assuming that the focal lengths are chosen so that the end of the depth of field for facet 1 coincides with the beginning of the depth of field for facet 2.

Thus, with only two focal planes, the holographic disc can double the depth of field of a conventional, nonholographic scanner.

If the disc is designed so that all of the facets are focused at different distances, then a much larger overall depth of field can be achieved. For example, if the minimum bar width 


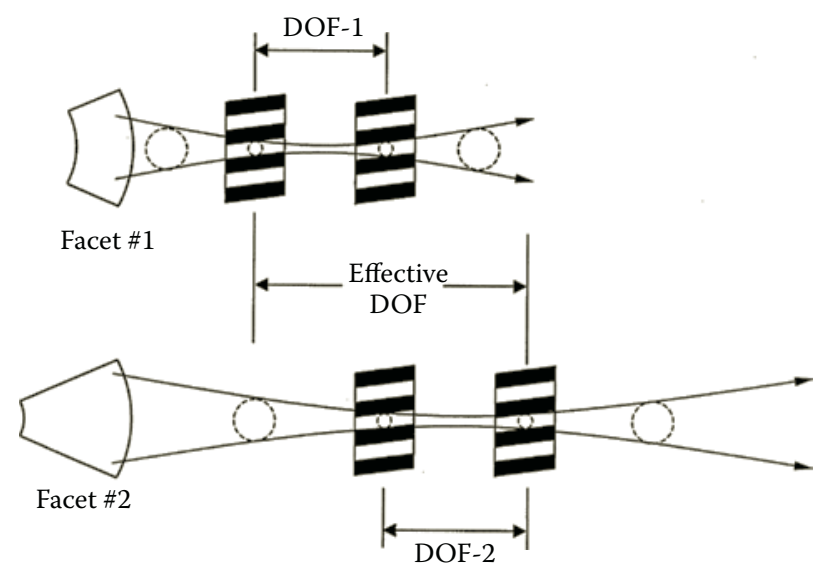

FIGURE 10.15

Combined depth of field for two holographic facets.

to be scanned is $0.2 \mathrm{~mm}$ (8 mils), a conventional, single-focal-length scanner would have a depth of field of approximately $200 \mathrm{~mm}$ (8 in). However, a properly designed holographic scanner could provide as much as $800 \mathrm{~mm}$ (32 in) depth of field for the same $0.2 \mathrm{~mm}$ minimum bar width code by using only four facets.

Even greater depth of field can be achieved with more facets; however, there are diminishing returns to this route to greater depth of field. Ideally, we would like to be able to control the focused spot size in each focal plane; however, this would require automatic aperture adjustment of the outgoing beam (similar to autofocus), which would prove mechanically difficult and very costly. As a compromise, typically, the centermost focal plane is optimized for maximum depth of field, which causes the other focal planes to be close to optimum as well.

Another limit to the continuing growth of depth of field is the very large distances that are eventually encountered as facet focal lengths increase. While theoretically this is not a problem for the resolution of the outgoing beam profile, it does present a greater challenge to light collection, which will be discussed later.

\subsection{OTHER FEATURES OF HOLOGRAPHIC SCANNING}

There are other novel features of holographic scanning that are not as obvious as the ability to provide a large depth of field. The major features of holographic scanning are:

1. Multiple focal planes

2. Overlapping focal zones

3. Variable light-collection aperture

4. Facet identification and scan tracking

5. Scan-angle magnification 
We have already discussed the multiple-focal-plane feature and the large depth of field that it provides. Let us now examine the other features to see what they are, how they are produced by the holographic disc, and what capability they provide.

\subsubsection{Overlapping Focal Zones}

We showed in the previous section how holographic scanning can provide a large depth of field by designing the holographic disc so that the depth-of-field region of each successive facet was contiguous with the depth-of-field regions of the facets immediately preceding it and following it. This may not, in practice, be the best disc design. It may, in fact, be better to design the holographic disc so that the focal point of one facet coincides with the limit of the depth of field for the preceding and following facets, resulting in an overlapping focal zone design. The reason why this design may be superior is explained in the following paragraphs.

One of the major contributors to decoding problems in a barcode scanner is the existence, or creation, of noise in the so-called quiet zone, the white, or clear, region immediately preceding and following the barcode. One of the contributing factors to noise in this region, and throughout the barcode, is substrate noise, or paper noise. Paper noise occurs when the size of the focused spot of the scanning laser beam is about the same as the size of the granularity of the substrate material. Paper fibers, for example, can be as large as $0.1 \mathrm{~mm}$ (4 mils). For very coarse paper or cardboard, the fiber size can be even greater. For nonpaper substrates, such as for barcodes etched into plastic or metal, the granularity can be greater still.

If a barcode on a noisy substrate is scanned at the focal point of a scanning laser beam, the small, in-focus spot will "see" the granularity of the substrate material. This will introduce paper noise on the return light signal that will, in turn, lower the probability of achieving a successful read.

While noise could, in general, be reduced with low-pass electrical filtering, the filter properties would have to be altered for each facet to correct for the differences in spot velocity. That is, a low-pass filter designed to remove noise from the short-focal-length facets would, at the same time, filter out the barcode signals from long-focal-length facets. Electrical filtering does not appear to be a practical solution for large depth-of-field scanners.

The solution to this problem is to scan the noisy barcode with a slightly out-of-focus spot. This spot will be larger than the in-focus spot, but still small enough to read the barcode. This larger spot will, while scanning the barcode, act as a filter to smooth out the surface roughness, effectively lowering the paper noise and increasing the probability of achieving a successful read.

Figure 10.16 shows the analog photodetector signal for a noisy barcode when scanned (a) by an in-focus spot, and (b) by a slightly out-of-focus spot. The noise on the signal from the in-focus scanning spot, is apparent. The resultant reduction in the noise level due to scanning the same barcode with a slightly out-of-focus scanning spot is equally apparent.

By overlapping the focal zones of the individual holographic facets, as shown in Figure 10.17 , we can guarantee that all barcodes will be scanned by both an in-focus scanning spot and one or more slightly out-of-focus scanning spots. The slightly out-of-focus spots will be small enough to read the barcodes, but large enough to smooth out the substrate noise.

This in-focus/out-of-focus capability, which would be difficult to implement with conventional scanning technology, is relatively simple to introduce with holographic scanning. One merely selects, during the master holographic disc design phase, the focal length for each of the facets that guarantees the desired amount of focal zone overlap. 


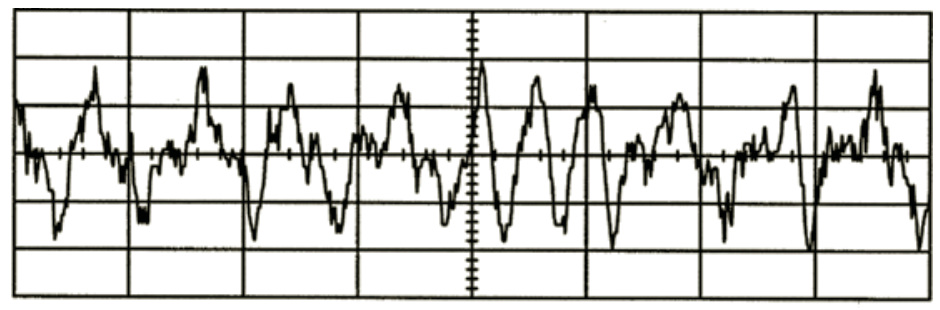

Scanning with in-focus spot

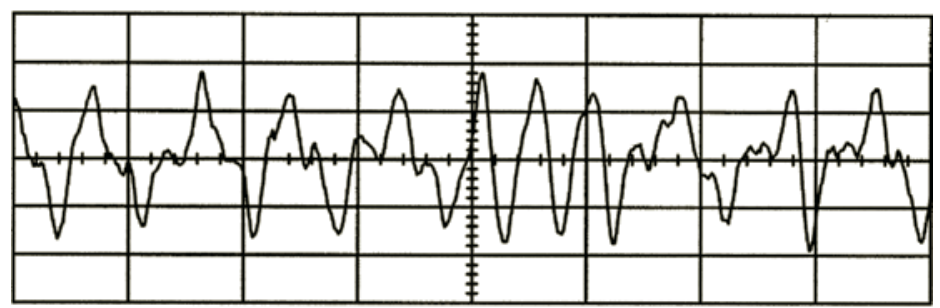

Scanning with out-of-focused spot

\section{FIGURE 10.16}

Photodetector signals for in-focus and out-of-focus scanning laser beams.

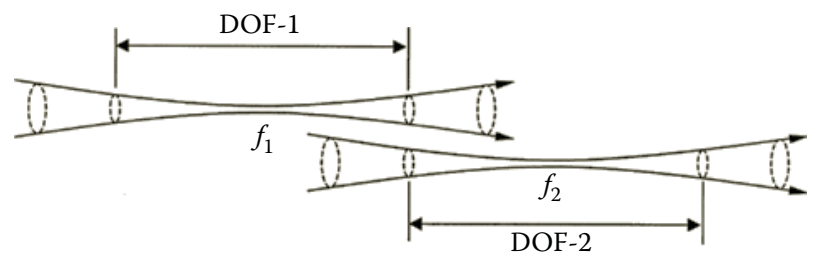

No overlap

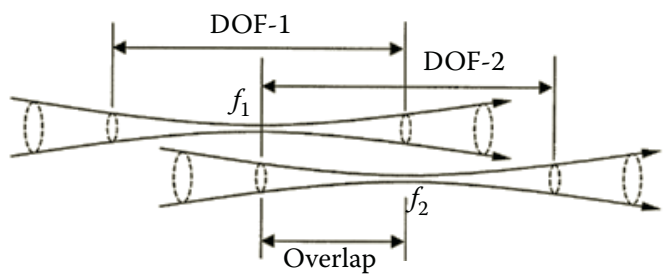

FIGURE 10.17

Overlapping focal zones of two holographic facets.

\subsubsection{Variable Light-Collection Aperture}

There is more to successfully achieving a large depth-of-field scanner than simply providing multiple focal planes. If, for example, one designs a scanner with a 1-m depth of field where the optical throw (closest reading distance) is $200 \mathrm{~mm}$ (8 in) and the range (farthest reading distance) is $1200 \mathrm{~mm}$ (47 in), then the variation in the light level returned to the detector, for barcodes with identical reflection characteristics, will be 36:1, the square of the ratio of the far and near distances. This places a severe dynamic range requirement on the analog electronics in the scanner. The problem is worse in practice since other factors, such as label skew and label reflectivity variations, also affect the amount of light returned. 
In order to reduce the variation in the light level of the return light in a multiple-focalplane scanning system, it would be desirable to vary the light-collection aperture to compensate for changes in the distance to the barcode being scanned. One could then use a relatively small aperture for a near-focus scan line and a relatively larger aperture for a far-focus scan line.

Holographic scanning allows one to do exactly that. Figure 10.18 shows a holographic scanning disc designed for focus distances ranging from 1000 to $1680 \mathrm{~mm}$ (39.5 to 66 in). Notice that the light collection area of each facet is different. The facet with the shortest focal length has the smallest light collection area while the facet with the longest focal length has the largest light collection area. The light collection area of each of the remaining intermediate facets is a direct function of its focal length.

This difference in light collection area for the near and far facets of the holographic disc allows the light collection to be approximately uniform over the total depth of field of the scanner. This is a major advantage in obtaining decoding accuracy over a large depth of field for a barcode scanning system.

\subsubsection{Facet Identification and Scan Tracking}

Note that the disc design shown in Figure 10.18 incorporates a gap in the outer annulus between two of the holographic facets. This gap may be transparent or opaque, depending on the application, and is referred to as the home-pulse gap. Because the outgoing laser beam is incident on the disc in this outer section, a detector placed in the proper location above the disc can sense this gap by measuring the laser power incident on the detector. As the gap passes over the laser the change in power recognized by the detector generates a home pulse in the analog signal. With this information embedded in the signal we can determine the rotational speed of the disc and, thereby, where on the disc the laser beam is currently incident. This knowledge can then be used to determine what facet is currently scanning, and even where in that facet the beam is located. This method of facet

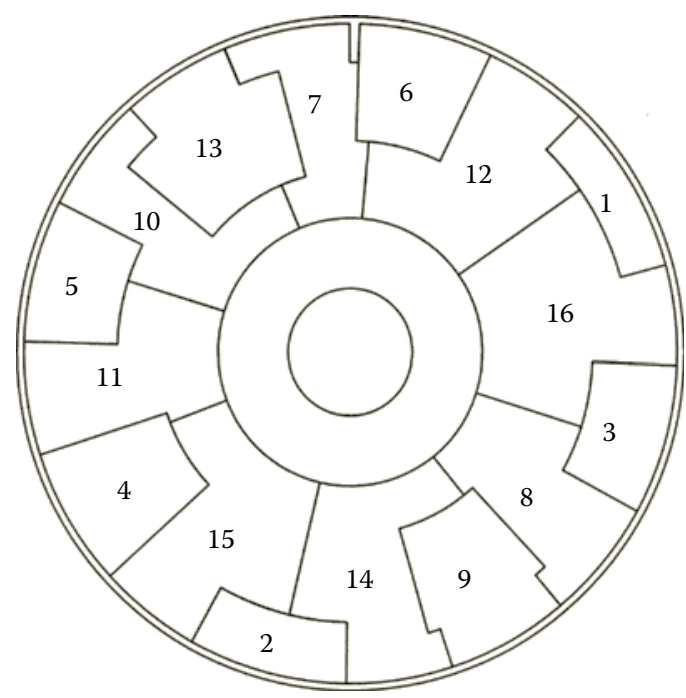

FIGURE 10.18

The Metrologie Penta holographic scanning disc showing large variation in facet areas. 
identification can be used in several ways to improve the decoding accuracy of the scanner, as well as provide for additional features.

If we knew, for example, that we were on a short-focus facet, we could decrease the electrical gain in the analog electronics. If we were on a long-focus facet, we could increase the gain of the analog electronics. This electronic automatic gain control (AGC) would add to the already existing optical AGC, introduced by the variable light-collection aperture, to further improve the decoding accuracy.

We could also vary the internal clock rate from facet to facet to improve resolution. Because the scanner is an angular scanner, the linear velocity of the scanning beam will vary directly with the distance from the scanner. The bit rate seen by the detector while scanning with a long-focus facet would be greater than the bit rate seen when scanning with a short-focus facet, assuming that the code density is the same in both cases. By making the clock rate vary from facet to facet to maintain the optimum clock rate for a given bit rate, we could, once again, improve the decoding accuracy of the scanner.

An additional capability made possible by this facet identification feature is scan tracking, for which several patents have been filed by Metrologie Instruments, Blackwood, NJ (U.S. patent numbers 6,382,515 B1; 6,457,642 B1; 6,517,004 B2; and 6,554,189 B1). Knowing precisely where the incident beam is striking each facet at each instant in time allows indirect determination of where the item being scanned is located in a given three-dimensional, spatial reference system. This knowledge is extremely useful in fully automated systems where little human interaction is desired, such as in scan tunnels in bulk-shipping centers. With the location of a scanned package identified, other automated mechanical systems can then redirect packages to their intended destination.

The reason holography lends itself so well to scan tracking is that the facets on a holographic disc are easily repeatable in the manufacturing process with high precision. Obtaining good repeatability of the deflector with mirrored scanning systems is more difficult.

\subsubsection{Scan-Angle Multiplication}

Holographic scanning discs used in barcode scanners are frequently designed to be illuminated with a collimated beam incident normal to the surface of the holographic disc. This illumination geometry provides a scanning spot that is free from aberrations across the entire scan line because the relative incidence geometry with respect to the hologram does not change as the disc rotates. This is a special case of the more general aberrationfree illumination geometry for rotationally symmetric systems in which the designed illumination beam is a converging spherical wavefront, converging toward a point located on the rotational axis of the disc. (A normally incident collimated beam has a point of convergence on the axis at infinity.) Under these conditions, the illuminating wavefront remains unchanged with respect to the hologram, always converging to the hologram's design convergence point even as the disc is rotated. Because the HOE was designed to produce an aberration-free diffracted beam when the incidence wavefront converges to that point, the diffracted beam remains aberration-free throughout the motion of the hologram. In essence, the playback (illumination) beam remains identical to the reference (recording) beam, which is the condition for zero aberrations.

If the holographic disc is designed to be illuminated with a collimated beam inclined at a non-normal angle, then some amount of aberrations will be introduced in the scanning beam. Each facet of the disc can be designed to still provide zero aberrations at the center of its corresponding scan line, but there will always be aberrations introduced as the disc 
rotates because of the resulting mismatch between the recording and playback wavefronts. The amount of the aberrations will be dependent on the amount of rotation away from the center of the facet and the amount of tilt of the collimated incident beam.

There is, however, one advantage to tilting the incident beam. It can be shown that a tilted, collimated reference beam will provide a greater scan-angle multiplication factor relative to an untilted, collimated reference beam geometry. A precise determination of the multiplication factor requires the use of a computer program because of the interdependence of the diffracted beam elevation angle ( $\beta$ in Figure 10.19) and the rotation angle $\left(\phi_{\text {rot }}\right)$. A first-order approximation, accurate to a few percent for holograms whose construction geometry is not too extreme, can be obtained from the following simple relationship. The variable $f$ represents the focal length of the holographic lens (facet). The other terms and the geometry are defined in Figure 10.19.

$$
f_{\mathrm{scan}}=f_{\mathrm{rot}}\left(\frac{r}{f} \cos g+\cos a+\cos b\right)=f_{\mathrm{rot}}\left(\frac{r}{f} \cos g+\frac{I}{d}\right)
$$

where $d$ represents the grating spacing of the hologram and the angle $\gamma$ is determined from Equation 10.9:

$$
\sin g=\cos b \sin q_{\mathrm{skew}}
$$

The multiplication effect of the tilted reference beam is due to the $\cos \alpha$ term in Equation 10.8; for normal incidence this term goes to zero.

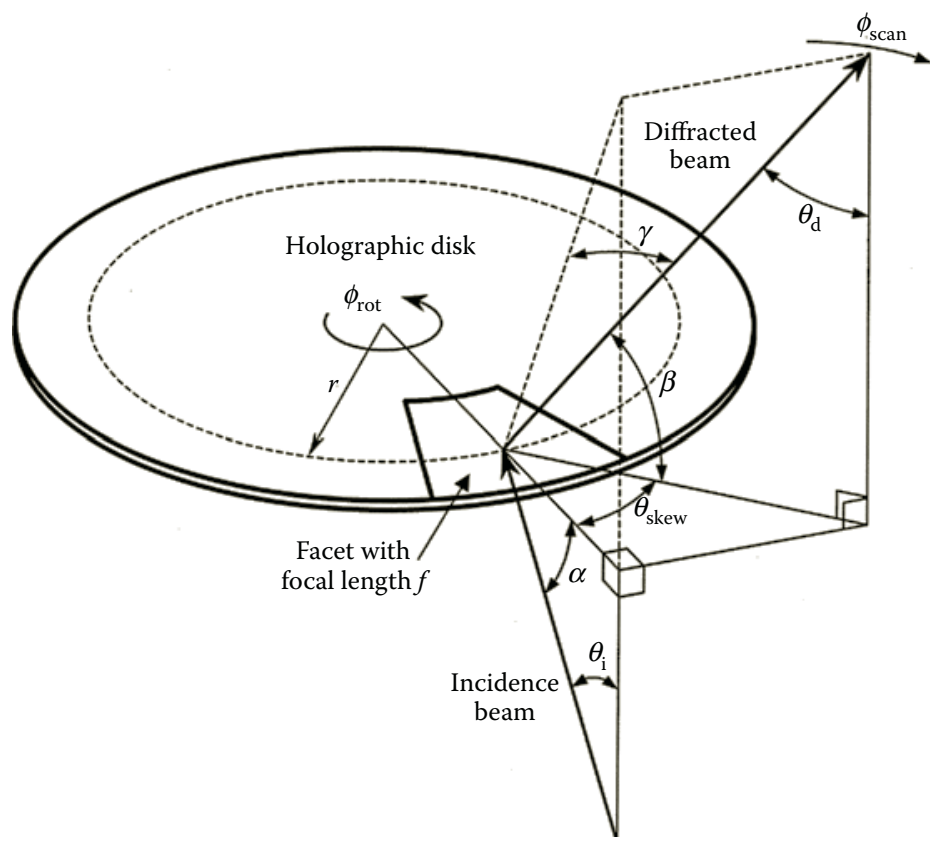

FIGURE 10.19

Scan-angle magnification parameters. 
As an example of the effect of the tilted reference beam, consider a holographic facet with $f=350 \mathrm{~mm}$ (13.8 in), $\theta_{\text {skew }}=40^{\circ}, \beta=66^{\circ}$, and $r=72 \mathrm{~mm}$ (2.8 in). For a normal reference (and incidence) beam $\left(\alpha=90^{\circ}\right), \phi_{\text {scan }}=0.605 \phi_{\text {rot }}$, while for a tilted reference resulting in an incident beam tilted at an angle of $22^{\circ}$ relative to the normal $\left(\alpha=68^{\circ}\right.$ in Figure 10.19), $\phi_{\text {scan }}$ $=0.980 \varnothing_{\text {rot }}$. The relative multiplication factor obtained by using the tilted incident beam is 1.62. This is a significant amount of scan-angle multiplication.

Scan-angle multiplication factors of this magnitude provide significant design flexibility. For a given scan length one could: make the disc smaller to produce a more compact unit; keep the disc the same size and add facets to provide more scan lines or to generate a more complex scan pattern; move the disc closer to the window to increase the light collection efficiency of the individual facets; or some combination of all three options. One may, of course, elect to use scan-angle magnification to just generate a longer scan line.

The incident-beam tilt angle, the aberrations, and the scan-angle multiplication factor are interrelated: the smaller the amount of tilt of a collimated incident beam, the smaller the aberrations; conversely, the larger the tilt, the larger the scan-angle multiplication factor. By careful selection of the tilt angle of the incident beam, one can get a significant amount of scan-angle multiplication while maintaining an acceptable amount of aberrations. The amount of aberrations introduced can be determined with a suitable ray tracing program. ${ }^{11}$ Acceptable levels of aberrations are established by the individual application.

If, on the other hand, the incidence angle is dictated by some other mechanical constraint in the scanner system, the aberrations can be controlled, as alluded to before, by controlling the designed convergence of the incidence beam. The closer the convergence point is to lying on the axis of disc rotation, the less severe the aberrations will be. This, however, has the same trade-off as manipulating the incidence angle. As the convergence of the incidence beam becomes shorter, the focal length, $f$, of the facet will become longer, perhaps even reaching or "passing" infinity and becoming negative. As this happens, the first term in Equation 10.8 gets smaller or, in the case of negative $f$, becomes negative, thereby canceling out some of the scan multiplication factor of the other two terms. Whether or not this is a disadvantage depends on what is more important to the application, scan-line length or spot quality.

\subsection{HOLOGRAPHIC DEFLECTOR MEDIA FOR HOLOGRAPHIC BARCODE SCANNERS}

All holographic barcode scanners today (2004) use a rotating circular disc as the substrate for the recording medium. Other geometries have been considered, but, for barcode reading applications, the disc geometry offers a number of manufacturing advantages and is generally less expensive.

All of today's holographic barcode readers also operate only in the transmission mode. It would not be impossible to develop a reflective holographic barcode scanner, but the transmission mode provides a simpler design, an easier manufacturing process, and less susceptibility to disc wobble. ${ }^{8}$

There are two general types of media suitable for recording HOEs on a disc surface for use in a holographic barcode scanner: surface-relief phase media, such as photoresist, and volume-phase media, such as bleached silver halide and dichromated gelatin (DCG). There are advantages and disadvantages associated with both types of media. For a 
more general review of the wide variety of holographic recording materials, see work by Bartolini $^{14}$ and Smith. ${ }^{15}$

The major factors influencing the selection of the type of holographic medium are manufacturing cost, diffraction efficiency, and, as will be described, the scan pattern density.

\subsubsection{Surface Relief Phase Media}

There are only two significant surface-relief phase media presently being used for holographic scanning discs-photoresist, and plastic copies made either directly from photoresist or from intermediate copies of the photoresist. As will be discussed later, this latter type is probably the least expensive of all to manufacture in a high-volume process and, from a purely cost consideration, this medium would appear to be the best choice for a holographic deflector disc.

The major disadvantage of the low-cost surface-relief material is that when using a simple mechanical replication process the resulting diffraction efficiency will be relatively low, on the order of $30 \%$. This is because a much larger grating aspect ratio (depth vs. spacing) is required to produce high efficiency. Mechanically releasing such a high-efficiency copy from the master can be very difficult, often damaging the master and the copy. This is a major drawback in barcode scanner applications. The low efficiency means that a higher power laser must be used to get sufficient laser power onto the barcode symbol to obtain a good reading. The greater cost of the higher power laser may offset the lower cost of the disc.

It is possible to get high diffraction efficiency using a surface-relief medium for light that is incident on the disk in an S-polarized orientation. ${ }^{16,17}$ It is not possible at the present time, however, to mechanically replicate these high-efficiency surface-relief holograms because of the aforementioned aspect ratio of the relief profile. (An example of such a high aspect ratio is shown in Figure 10.20.) This means that original holograms, not inexpensive copies, would have to be used. In some holographic deflector applications, this is acceptable. In general, however, this is not an acceptable alternative for a holographic barcode scanner due to the higher cost of the discs and their greater susceptibility to physical damage. (Surface relief holographic discs cannot be protected by a cover glass since an index-matching adhesive would effectively eliminate the surface relief structure.)

The low diffraction efficiency of the mechanically replicated surface-relief material also means that the collected light will be low in a system employing the holographic facets of the disc in a retroreflective mode. This loss in collected light cannot be compensated by increasing the laser power further because of the limitations established by the federal laser safety standards. The only means left to compensate for the low diffraction efficiency in the collected light is to increase the size of the facets on the holographic disc. However, this reduces the total number of facets, hence the total number of independent scan lines, and the subsequent scan pattern density. In some barcode scanning applications, this may be an acceptable trade-off. In other applications, such a trade-off may be unacceptable.

For example, in supermarket/retail barcode scanning applications, the depth-of-field requirement is relatively moderate, so that a holographic scanner with as few as two focal planes can provide adequate performance. However, in industrial barcode scanning applications, the required depth of field may be as large as $1 \mathrm{~m}$ (40 in), or more, for medium density barcodes (barcodes with a minimum bar width on the order of $0.3 \mathrm{~mm}$ ). This kind of depth-of-field requirement can only be met with a scanner that can provide a large number of focal planes. A holographic scanner can be designed to provide this capability, but the number of independent facets on the scanning disc must be as large as possible. 


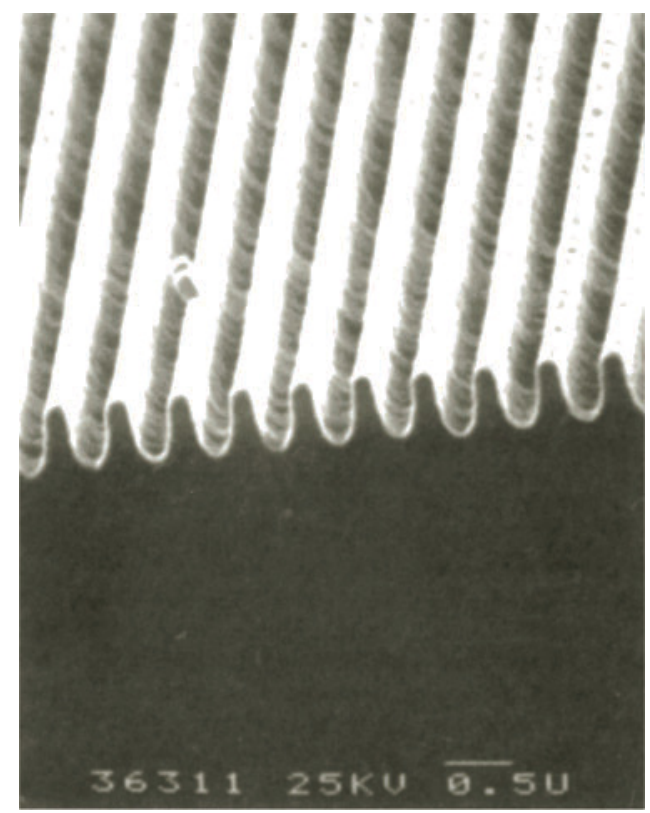

FIGURE 10.20

Surface-relief hologram in positive photoresist.

Therefore, any reduction in the number of facets imposed by a low diffraction efficiency recording medium will reduce the depth of field.

For many retrocollection scanning applications even the "high-efficiency" holograms mentioned above do not have a high enough diffraction efficiency for good light collection. In order to eliminate specular reflection noise in a barcode scanner, a polarizer is often placed in front of the light detector. This is done so that one linear light polarization can be used for the outgoing, scanning beam while the other, perpendicular light polarization will return to the scanner, pass through the polarizer, and be detected. This presents a problem for surface-relief holograms in that an even higher aspect-ratio relief profile is necessary to diffract P-polarized light than is necessary to diffract S-polarized light. As a result, while the outgoing efficiency could produce a strong scanning beam, the light collection ability would be relatively weak (or vice versa) requiring the same kind of tradeoffs as discussed above.

Even so, despite the relatively low diffraction efficiency of the holographic discs produced by mechanical replication, which is a surface relief process, the low cost of such discs make them very attractive in supermarket and retail barcode scanners in which component cost is a major factor.

\subsubsection{Volume Phase Media}

Volume phase materials are capable of very high diffraction efficiencies, on the order of $90 \%$ or more. Such high efficiencies means that the individual facets on the disc can be relatively small, even when the disc is used in a retroreflective mode. This means that there can be more facets on the disc, which, in turn, means that the scanner can generate more independent scan lines, resulting in a larger depth of field and/or a more complex 
scan pattern. The higher diffraction efficiency also means that a lower power laser can be used to generate the scan lines.

There are a number of materials that are suitable for use as volume phase materials in holographic scanners. The first material that comes to mind is bleached silver halide. In this process, the absorptive structure in a photographic emulsion hologram is chemically converted from metallic silver to a material having a refractive index different than the surrounding gelatin matrix. ${ }^{18-21}$ For example, the silver may be rehalogenated by exposure to bromine vapor. Holograms created with this material can have high diffraction efficiencies, on the order of $80 \%$ or more. ${ }^{22,23}$ Processing is relatively simple, and the holograms are reasonably stable. There are a few bleaches, however, that leave reaction products behind in the emulsion. Some of these products are photosensitive and exhibit printout effects, particularly when subjected to intense ultraviolet irradiation. Nevertheless, moderately efficient holograms can be realized, and the advantages of photographic emulsions, such as extended spectral response and speed, may be exploited. One practical disadvantage associated with this material is that the discs must be coated and sensitized by one of the major companies producing general photographic materials. Such companies are usually reluctant to stock odd substrate shapes (like discs) and coat them to a user's specifications, in quantities that are, for them, relatively small. This creates a very real sourcing problem.

The next most attractive volume phase material is photopolymer. ${ }^{24,25}$ Cross-linking in these materials is produced when they are exposed to light of relatively short wavelength, blue to ultraviolet. When a photopolymer is exposed to a holographic fringe pattern at the proper wavelength, the periodic variation in light intensity of the fringe pattern produces a corresponding periodic variation in cross-linking in the polymer. When developed, the photopolymer will exhibit a periodic variation in refractive index corresponding to this periodic variation in cross-linking. These materials are relatively stable when exposed to normal levels of ambient light, heat, and humidity.

The main drawback to these materials has been their relatively small change in refractive index, $\Delta n$, produced by exposure to light and subsequent processing. This means that, in order to get high diffraction efficiency, the thickness of the photopolymer coating has to be on the order of 50 microns (2 mils). Such a large thickness would make the holographic deflector disc very sensitive to the Bragg angle. That is, very slight deviations in the angle of incidence of the reconstruction beam in the scanner would cause severe reductions in the disc diffraction efficiency. Deviations on the order of $1 / 4^{\circ}(4.4 \mathrm{mrad})$ could cut the diffraction efficiency in half. ${ }^{26}$ This is generally unacceptable in a product where the total angular manufacturing tolerances could easily be this large. Furthermore, the anticipated mode of operation could cause the effective angle of incidence to vary by $1 / 4^{\circ}(4.4 \mathrm{mrad})$ during disc rotation.

The DuPont ${ }^{27}$ and Polaroid ${ }^{28}$ photopolymers have exhibited refractive index changes that are much greater than those of previous photopolymers. $\Delta n$ values approaching the values obtainable with DCG (nearly ten times as great as earlier photopolymer $\Delta n$ values) have been obtained. These materials have great potential for use as a recording medium for holographic deflectors used in barcode scanners, since high diffraction efficiency should be achievable in relatively thin coatings, on the order of 5 microns ( $200 \mu \mathrm{in})$.

The volume phase material that has, up to now, been the most successful material for use in holographic deflectors for barcode scanners is DCG ${ }^{29-31}$ The major advantage of this material, as a medium for holographic deflector discs, is that its diffraction efficiency can be very high $(>90 \%)$ in a relatively thin (3-5 microns or 120-200 $\mu$ in) coating because of its high $\Delta n(0.10-0.15$ or greater). This means that DCG can have, simultaneously, high diffraction efficiency and very low Bragg-angle sensitivity. This is a significant advantage from both a manufacturing and an application standpoint. 
The major disadvantage of DCG is that it is extremely sensitive to moisture. Holograms made with DCG must be sealed to protect them from environmental moisture.

From the standpoint of the development of a barcode scanner, there is one other disadvantage to DCG. Although it has been around a long time, DCG is the least understood of all the holographic recording media. There are at least three theories that claim to explain the mechanism of image formation, ${ }^{32-34}$ and there are as many recipes for processing DCG as there are authors writing on the subject. Many of them start with gelatin that is already coated on photographic plates, ${ }^{35}$ a procedure which is unacceptable for the same reasons that bleached silver halide is unacceptable: the sourcing problem.

In most large corporations, one will also find considerable resistance to the use of DCG. Most chemists feel comfortable with well-understood inorganic materials, such as silicon, and the more traditional organics, such as photoresist, photopolymer, and so on. DCG is an organic material whose properties are poorly understood and relatively unpredictable. Gelatin is, after all, made from the skins, bones, and connective tissues of animals. Its properties can vary depending on what the animals ate or where they were raised.

Nevertheless, because of its excellent holographic qualities, DCG is one of the best recording materials for holographic deflectors used in barcode scanners. It is relatively stable when exposed to normal ambient temperatures. However, it is extremely moisture sensitive and must be sealed to protect it from normal ambient humidity.

DCG is generally sensitive only to the short wavelength portion of the visible spectrum, $\lambda<520 \mathrm{~nm}(20.5 \mu \mathrm{in})$, and although it is possible to sensitize it to the red end of the spectrum $^{36-40}$ only moderate success has been achieved. The primary problem has been removal of the residual sensitizing dye to give a complete phase structure. For barcode scanning applications, where the light source in the scanner is generally a visible laser diode (VLD) with a wavelength somewhere in the red region of the spectrum, unsensitized DCG cannot be used to make the master holograms at the operating wavelength. Because of this, the DCG holographic disc must be made as a copy of masters formed in one of two ways.

In the first method, the wavelength that will be used in the scanner is used to construct the masters with a material that is sensitive to that region of the spectrum, such as silver halide. This allows a relatively simple optical setup that will produce an aberrationfree hologram. Typically, DCG submasters (in the form of a submaster disc, or individual submasters) are made from the masters, providing greater efficiency to the production process.

In the second method, the masters are made directly in DCG using a wavelength within its spectral sensitivity range, such as $488 \mathrm{~nm}(19.2 \mu \mathrm{in})$, one of the high-power lines of an argon laser. The difference of the exposure wavelength from that which the scanner will employ requires some kind of aberration-compensating optics in the master-exposure setup. This makes the setup more complicated, but the result can be a higher-quality hologram since the submaster step of the process is not needed, and the use of additional aberration-correcting optics will maintain the essentially aberration-free performance of the final hologram.

Whichever method is used, the DCG copy disc can then be made using any wavelength to which the DCG is sensitive. There will be no aberrations introduced in the copy process, regardless of the wavelength used in the copy process. We will have more to say about this in the section on disc fabrication.

DCG is processed in a sequence of alcohol/water baths of varying concentrations of alcohol and varying temperatures. Times, temperatures, and concentrations vary, depending on whose process is used. 
Diffraction efficiencies obtained with DCG approach the theoretical limits for volume phase materials. Efficiencies greater than $90 \%$ can be readily obtained. The only things limiting the diffraction efficiencies of a sealed DCG holographic disc are reflections off the glass surfaces and absorption and scattering losses in the gelatin. If antireflection (AR) coatings are not employed, the Fresnel reflections at the air/glass interfaces will cause the primary losses. This may limit maximum efficiency to about $70 \%$, depending on polarization. If, however, good AR coatings can be applied, there will still be some minor Fresnel losses at the internal gelatin/glass interface and some small amount of scattering and absorption in the gelatin. If the film properties can be controlled enough to keep scattering to a minimum, then efficiencies of $95 \%$ are achievable. This control, however, is the key, and is sometimes easier said than done. Even so, efficiencies in excess of $85 \%$ are relatively easy to maintain.

\subsection{FABRICATION OF HOLOGRAPHIC DEFLECTORS}

\subsubsection{The DCG Holographic Disc}

One possible DCG holographic disc fabrication process using DCG masters (no submasters) is shown schematically in Figure 10.21. Each facet of the holographic master disc is recorded individually using an argon laser and a vibration-isolated table. The facets are recorded on rectangular DCG holographic plates, which are then processed in the water/ alcohol baths. When a suitable efficiency is achieved, resulting in an optimum intensity ratio of diffracted to transmitted light, the facets are "capped" (sealed with another piece of glass using an index-matching optical adhesive) for moisture protection then cut to a size appropriate to an automated copy-exposure machine. They are then masked to provide the designed size and shape when exposed by the copy beam.

The DCG copy disc fabrication process resembles, but is not identical to, a photographic contact-copy process. All of the DCG masters are placed on a computer-controlled wheel in the appropriate sequence. When the exposure cycle is started, a DCG disc is brought in under the master wheel with a slight air gap between the disc and the master. Because of this gap the process is not a true contact-copy process and some settling time is required after the motion stops before the exposure can begin. In situations where relative motion between the master and the disc is not required (such as with a submaster disc), index matching fluid can be used between the master and the disc. This limits relative motion and also greatly reduces reflections at the interface. For air gap copying, AR-coated caps are recommended for the masters. During the exposure sequence each master facet swings into position and is then sequentially illuminated in a step-and-repeat exposure process, using an expanded beam from an argon laser. That beam may be collimated, divergent, or convergent, depending on the desired characteristics of the copy HOE. The angle of illumination of the laser beam is modified from facet to facet considering each facet's construction and the difference between the copy-exposure wavelength and the scanner wavelength.

Exposure of each master holographic facet creates an optically identical holographic copy facet in the DCG through the interference of the diffracted beam with the undiffracted zero-order beam. As long as the copy process is reasonably close to a contact-copy process there will be no aberrations introduced by copying, regardless of copy wavelength or exposure angle. The reason for this is that the recorded interference pattern in the 


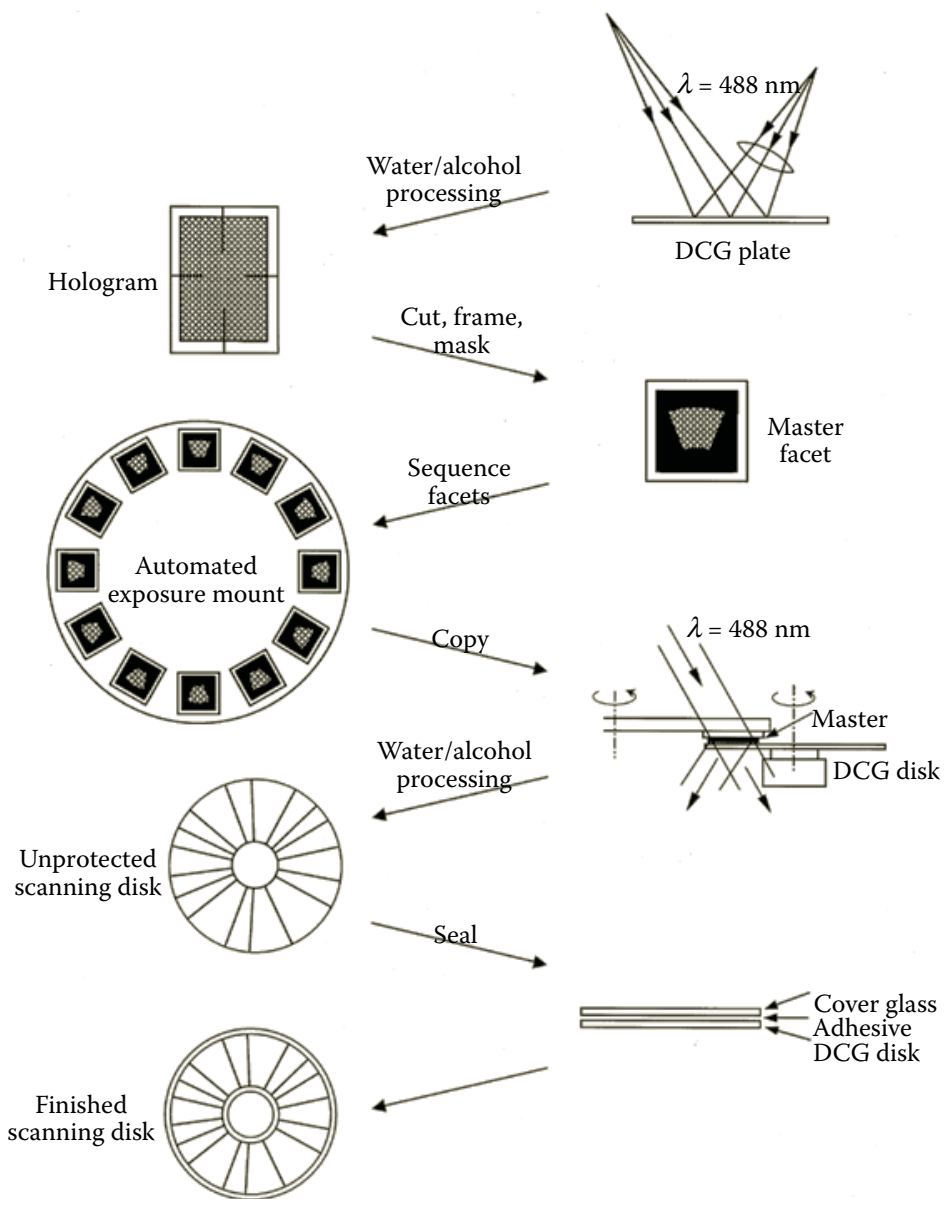

FIGURE 10.21

Holographie disc fabrication process.

hologram will accept any configuration of incidence beam and produce a corresponding conjugate diffraction beam. Because those two beams were created from that interference pattern, those two beams are the exact beams that will recreate that same interference pattern, provided the recording medium is located in the same place as the master hologram. The more removed the recording medium is from the master hologram, the more the properties of the two will differ. For a very small space, the difference is insignificant.

When all of the facets of the DCG copy disc are exposed, the disc is processed in a sequence of water and alcohol baths. The process is essentially the same as the process for the DCG masters, although some of the timing may be different, and precise control and consistency are, ironically, more important in the production process than they are in the master process. This is because DCG can be reprocessed if the initial results are not satisfactory; however, reprocessing leads to inconsistency and inefficiency, both of which are undesirable in a production environment. The details of the baths, the relative times, and the temperatures of the liquids, are proprietary.

A major objective in any DCG disc manufacturing operation is to establish a total exposure and development process that provides consistent results and high yield. One of the more difficult problems encountered in attempting to do this is the problem of "gel 
swell" - the tendency for the exposed and processed gelatin to be thicker than the unexposed, unprocessed gelatin. This residual gelatin swell causes a shifting of the Bragg planes within the thickness of the gelatin so that the angle of the Bragg planes, relative to the surface of the gelatin, is not the same after processing as it was during exposure (see Figure 10.22). This results in a decrease in diffraction efficiency when the reconstruction beam is at the designed incidence angle. Any attempt to increase the diffraction efficiency by changing the reconstruction beam angle will introduce undesirable aberrations.

A similar effect also occurs if the postprocessing bulk refractive index is different from that anticipated. A different refractive index causes the incident reconstruction beam to refract into the gelatin at an angle different from that expected and, thereby, meet the Bragg planes at the wrong angle. Because the bulk index in DCG is dependent on the amount of microscopic air voids present in the processed gelatin, the bulk index is sensitive to changes in both the film preparation process and the water/alcohol process. In preparation, if the film is excessively hardened it will be more difficult to form voids and the bulk index will be higher. In general this also limits the range of the index modulation, $\Delta n$. During wet processing, if the hologram is left too long in hot water the gel can be oversoftened, creating excessive voids and lowering the bulk index. This also can cause the problem of excess scattering losses since the voids can become larger and, therefore, make the gel appear less homogenous to the scanner wavelength.

Gel swell and bulk index changes are separate effects that can be separately measured; however, symptomatically they produce the same effect-reduction in efficiency due to "Bragg error." Several methods of eliminating these undesirable effects have been described in the literature. ${ }^{35}$ Generally, these involve either some sort of postprocessing chemical treatment or some form of postprocessing baking of the hologram. None of these methods is predictable enough to be suitable in a manufacturing process. If, however, the gel swell is predictable and consistent, it can be compensated for in the copy process by reducing the calculated angle of incidence of the argon laser copy beam. This increases the Bragg plane tilt angle. After processing, the gel swell will raise the Bragg planes, decreasing the tilt angle until it equals the original value. This process is described in greater detail by Dickson. ${ }^{42}$

If the processing methods are well controlled, so that the gel swell and bulk index are both predictable and consistent, then one can eliminate the Bragg error problem by altering the copy beam angle. Altering the copy beam angle, incidentally, has no effect on the optical properties of the holographic copy disc since these are fixed in the surface fringe

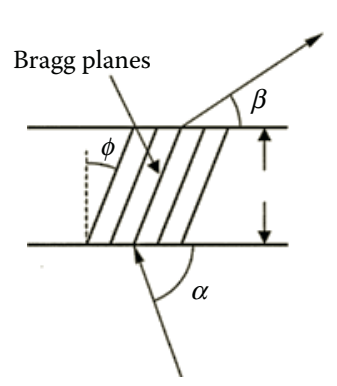

Exposure

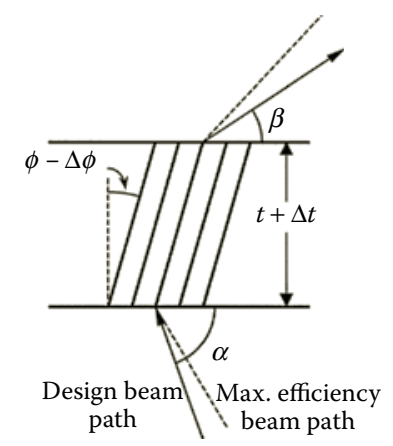

After processing

FIGURE 10.22

Effect of gel swell on the angle of the Bragg planes. 
structure of the master. The copy process will always faithfully reproduce this fringe structure.

After the DCG disc is exposed and processed, several millimeters of the gelatin are stripped from the outer and inner edges to inhibit wicking-in of moisture in the sealed disc. The disc is then sealed with a glass cover disc for protection from moisture, using an index-matching optical adhesive. A metal hub is then bonded to the inner diameter of the disc and the disc is dynamically balanced.

The optical properties of each and every DCG copy disc will be identical to those of the master holographic disc. The optical characteristics of the holographic scanner are essentially established at the time of the construction of the holographic master. While it is possible to modify these characteristics somewhat through variations in predisc or postdisc optics, this is generally not done in holographic barcode scanners.

\subsubsection{The Mechanically Replicated Surface-Relief Holographic Disc}

The other primary holographic recording material, photoresist, has a diffracting structure in the form of surface deformation or relief as shown in Figure 10.20.16,17 Consideration can therefore be given to using mechanical replication for mass production. This is not to imply that optical copying techniques cannot be used with surface relief, because they certainly can, either as master or copy or both.

Mechanical replication of surface relief is not new, having been developed decades ago for low-cost replication of mechanically ruled gratings. Today, it has become one of the primary manufacturing techniques of the production of high-quality gratings from holographic masters. ${ }^{43}$

Although replication of a surface relief hologram can be performed directly from the photoresist master, there is some danger that the photoresist may not stand up to repeated mechanical pressures, elevated temperatures, and/or the copy-master release process. Considering the difficulty and potential expense associated with master fabrication, a replication process that permits maximum replication volume is required. This is accomplished by the fabrication of a more durable submaster and usually results in the master itself being sacrificed. A very early technique, borrowed from the audiorecording industry uses a metal "stamper" to emboss or compression mold the relief into a vinyl thermoplastic. ${ }^{44,45}$

In the adaptation of this process, ${ }^{46,47}$ a very fine grain layer of nickel or gold is deposited by evaporation or sputtering onto the relief to form a conductive conformal coating, typically a few hundred Angstroms thick. Nickel formation is then continued by other methods, such as electrochemical deposition, until a thickness of several hundred microns is achieved. At this point, the outer nickel surface has no significant relief and it can be attached to a rigid substrate. The sandwich is separated at the nickel/resist interface and residual photoresist dissolved away leaving behind a rigid metal replication of the relief. This structure is a negative of the original and can be used in hot pressing, injection molding, or epoxy replication processes, which will be discussed later.

An alternative method of submaster preparation is to transfer the resist relief downward into its own substrate by radio frequency (RF) sputter etching or reactive ion etching (RIE) techniques. ${ }^{48,49}$ In these methods, the relief surface is removed at a uniform rate by bombarding the relief with accelerated ions or, in the case of RIE, with reactive atoms that react with the substrate molecules to form a volatile gas. The valleys of the resist pattern disappear first and the underlying substrate is exposed and etching occurs. By the time the resist peaks have disappeared, the valley areas are deeply cut into the substrate. 
Proper choice of photoresist, substrate materials (such as silicon or quartz), and plasma parameters allows the surface relief to be accurately transferred into the substrate and the cross-sectional shape to be preserved. ${ }^{50}$ These processes result in a submaster having a positive replication of the original master in contrast to the negative shape of the previous nickel submaster.

Once fabricated, these more durable submasters may be used to generate multiple copies. One such method, thermal mechanical embossing or compression molding, is accomplished by pressing the relief into a heated and softened thermoplastic film such as polymethyl methacrylate (PMMA) or polyvinyl chloride (PVC). Bartolini and colleagues ${ }^{46}$ rolled the submaster together with a vinyl strip between two heated cylinders. Gale et al. ${ }^{47}$ used a conventional hot stamping press at $150{ }^{\circ} \mathrm{C}$ and $3 \mathrm{~atm}$. to emboss into PVC sheets. A similar pressing technique was used by Iwata and $\mathrm{Tsujiuchi}^{51}$ with separation of the copy from the mold performed by sudden cooling and differential contraction.

Replication by pressing tends to introduce considerable strain and other inhomogeneities into the new substrate. These problems can be overcome by using injection molding techniques that have been developed for high-volume, high-quality fabrication of plastic lenses. ${ }^{52}$ In this case, the submaster is one surface and an optically polished stainless steel flat is used as the facing, parallel surface. The appropriate polymer is plasticized to a more fluid state than used by compression molding and introduced into the temperaturecontrolled mold under high pressure. ${ }^{53}$ Most of the materials in use are copolymers of PVC, polyvinyl acetate (PVA), and acrylic (PMMA) compounds. The acrylic material has an advantage over vinyl due to the lack of birefringence in the finished substrate, and the stability and ease with which it can be machined and polished.

The final alternative is to use a polymer that can be cross-linked by ultraviolet illumination. ${ }^{54}$ This technique eliminates the need for high-temperature processing and reduces the possibility of induced stresses and dimensional changes upon cooling/curing. An injection mold apparatus can be adapted for these purposes as long as one plate is sufficiently transparent for the UV illumination. Depending on the use of release agents and relative adhesion, the replica can also be attached directly to a rigid substrate in the same operation.

\subsection{AN EXAMPLE OF A HOLOGRAPHIC BARCODE SCANNER: THE METROLOGIC PENTA SCANNER}

As an example of a holographic scanner that uses most of the design techniques and methods discussed thus far we will now discuss the Metrologie Penta Scanner, an industrial application scanner that exploits many of the advantages of holography. First we will discuss the design of the scan pattern and then the means by which it is produced.

\subsubsection{The Penta Scan Pattern}

The Penta scanner was designed as a large-scale "pass-through" scanner. In general, it was designed to create an aggressive scan volume at some distance from the scanner through which packages would pass in a roughly uniform manner. Ideally, a large range of package sizes with barcodes of moderate resolution would be able to pass through the scan volume, either manually or automatically, and as long as the package was roughly facing the scanner it would be successfully scanned throughout a large depth of field. 
This broad application definition places several requirements on the design. A large depth of field will require multiple focal planes. With no specification given to package orientation the scanner will have to read essentially all orientations. Also, the variety of package sizes requires a large scan pattern size coming out of the scanner. All of these requirements can be met, quite easily, with holography.

The name of the "Penta" scanner comes from the pentagonal configuration of the scan pattern, shown in Figure 10.23. The basic pattern is formed by combining five simple rasters (groups of parallel lines) of different angular orientation. The rasters are evenly spaced through an entire $360^{\circ}$, making this pattern omnidirectional. Omnidirectionality is an essential characteristic for a barcode scanner if it is desired that the operator not waste time trying to identify the proper orientation of the code for a good scan. In automated applications, where the highest efficiency is desired, packages often are presented in truly random orientations as they pass under the scanner on a conveyor belt.

Assuming a direction of package flow, as shown in Figure 10.23, the primary parameters that define the pattern are the scan line length, the line separation, and the number of lines per orientation group. These parameters must be combined in such a way as to provide the desired total pattern omniwidth (width over which full omnidirectional scanning is possible). At the same time enough overlap in the near-vertical fields must be maintained such that codes in the "ladder" orientation (i.e., codes travelling in the direction perpendicular to its own bars and spaces) cannot slip through the pattern unscanned.

This pentagonal pattern not only provides for omnidirectional scanning, but it is also very aggressive on codes with higher aspect ratios, which are more difficult to scan than square codes like the original UPC code. The smaller angle between adjacent scan-line groups means each group has a smaller angular range that it must cover, and, therefore, less code height is required. This also means there will be less reliance on software stitching algorithms, but such algorithms can still be employed to make the scanner's performance even better.

Once the optimum scan pattern is established, the task still remains to provide for a large depth of field. Since the optimum performance pattern has been determined, the logical conclusion is to repeat that pattern several times at different distances from the scanner.

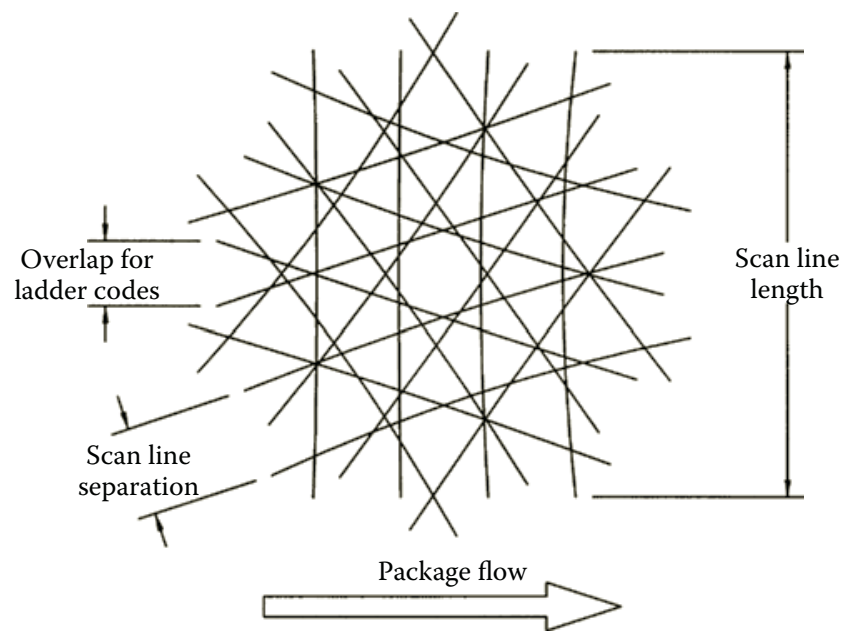

FIGURE 10.23

The two-dimensional representation of the Penta scan pattern. 

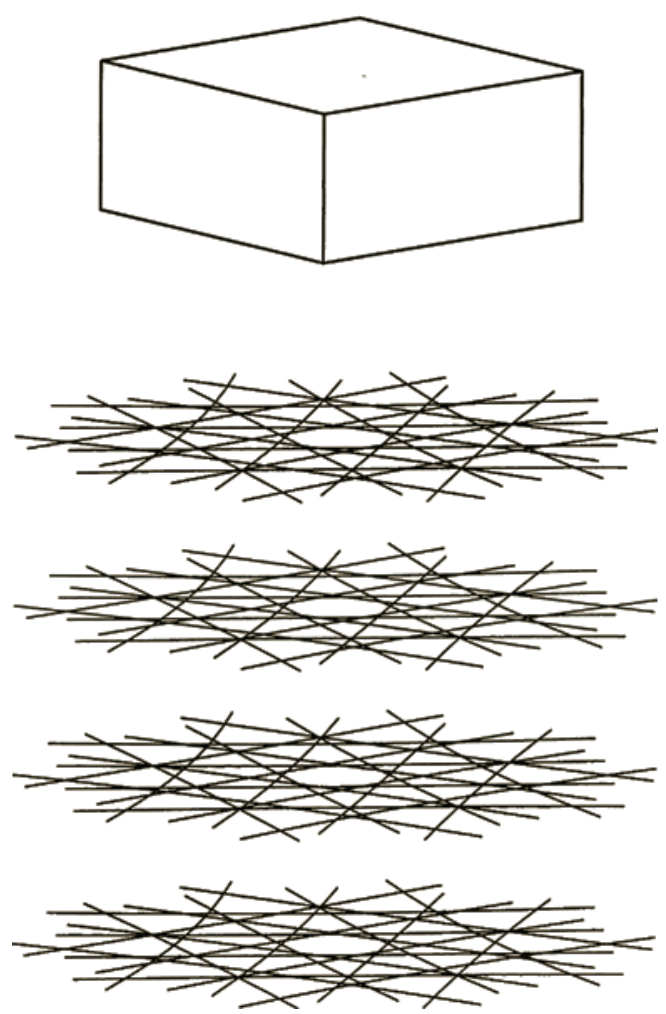

FIGURE 10.24

The three-dimensional scan volume of the Penta scanner.

Different focal planes are established providing acceptable overlap and producing a full, contiguous depth of field. For Penta four focal planes were chosen. A three-dimensional representation of the Penta scan pattern is shown in Figure 10.24.

\subsubsection{The Penta Scanning Mechanism}

The heart of the Penta scanning mechanism is, of course, a holographic disc. Also included are five scanning stations located around the periphery of the disc, each comprised of a VLD module prior to the disc and a folding mirror after the disc to direct the beams out the scanner window. A top view of the scanner with the cover removed, showing the five scanning stations, is shown in Figure 10.25.

A clearer view of the optics of a single scanning station can be seen from the side view in Figure 10.26. The dotted line in Figure 10.26 represents the outgoing beam path. The path starts at the VLD and is first roughly collimated by a conventional, aspheric lens. From there it reflects off a folding mirror, which directs the beam to the multifunction plate (MFP). The MFP is a multipurpose hologram, which, along with the VLD, lens, and mirror, finishes the subassembly of the "optics module."

The VLDs used in barcode scanners today have certain inherent properties, some of which are undesirable. With the use of the MFP, however, some of the undesirable effects can be alleviated. The functions performed by the MFP include beam aspect-ratio modification, astigmatism reduction, and dispersion minimization. In fact, VLDs inherently 


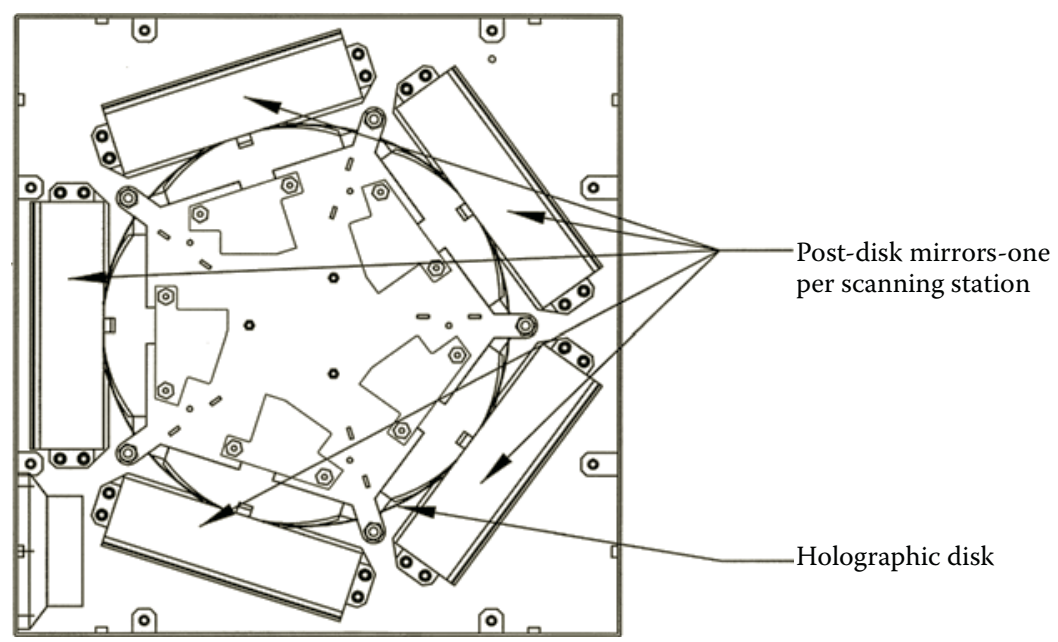

FIGURE 10.25

Penta scanner top view showing the different scanning stations.

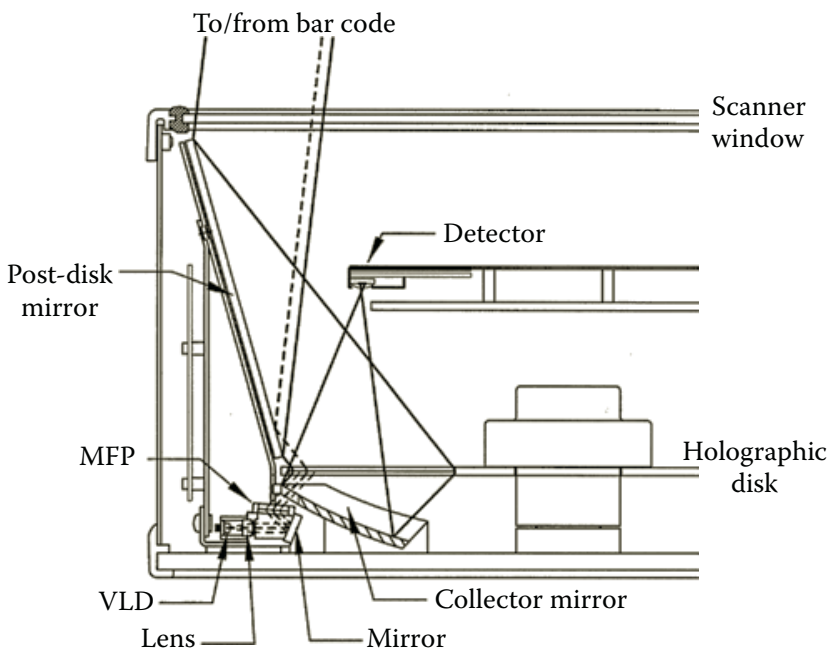

FIGURE 10.26

Penta scanner side view showing the scanning and light-collection optics.

produce beams with an elliptical shape and a characteristic astigmatism. Both of these properties of the beam can be manipulated by a single MFP (or by more than one if a greater range of control is desired) by simply choosing the incidence and diffraction angles of the MFP properly. At the same time, the dispersion produced by the facets of the disc (natural to all diffraction gratings) can be minimized by the same MFP by countering it with its own dispersion.

Following the MFP the beam heads directly to the holographic disc, incident on it at a specified angle. This is where the primary pattern formation occurs. Each of the five rasters in the pattern in Figure 10.23 contains four lines, and that pattern is repeated in four focal planes. This requires 16 unique facets on the disc, which were shown previously in 


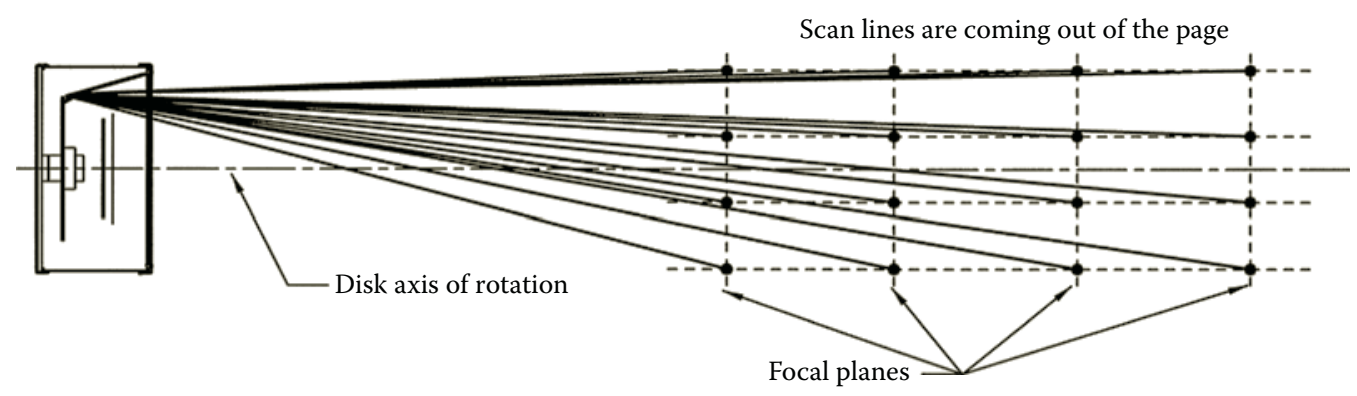

FIGURE 10.27

Side view of the scan lines produced by one scanning station.

Figure 10.18. Each one of these facets has a different diffraction angle, $\theta_{\mathrm{d}}$, and a different focal length, $f$, which results in the beam being focused at a different distance, $s$. The result of combining all the different focal lengths and diffraction angles is shown in Figure 10.27. Choosing the right combination of focal lengths and diffraction angles results in scan lines that are laterally equidistant from the disc rotation axis, thereby reproducing the desired pattern at the different distances.

The solid lines in Figure 10.26 represent the path of the light reflected off a barcode as it makes its way to the signal detector. The light that is collected returns essentially along the same path by which it left the scanner, making the scanner a retrocollective system. On the way back the light has diffusely spread out to completely fill the aperture of the facet, after first being reflected off the large pattern-folding mirror. The light is then diffracted back towards the module; however, a collector mirror is in the path everywhere except for a small hole through which the outgoing beam passed.

This collector mirror generally has a parabolic or elliptical shape. The light incident on the collector is focused and directed upward to the signal detector; however, in order to get there it must, once more, pass through the disc. On this third pass, however, the desire is for the disc to not affect the beam in any way. In reality it is impossible not to have some losses occur, but if the disc is correctly designed and manufactured those losses need not be much worse than that of a plain piece of glass. This is due to the angular sensitivity of the diffraction efficiency of the holograms. A properly manufactured disc will only have high efficiency at the designed incidence and diffraction angles. Because the rays proceeding from the collector to the detector are incident on the disc at angles far enough removed from the design incidence and diffraction angles, the resulting transmission of the disc is relatively high.

\section{REFERENCES}

1. Savir, D.; Laurer, D.J. IBM Systems J. 1975, 14, 16.

2. Broockman, E. U.S. Patent 4,717,818, assigned to IBM, January 5, 1988.

3. Allais, D.C. Bar Code Symbology; Intermec Corporation: Everett WA, 1984.

4. Cindrich, I. Appl. Opt. 1967, 6, 531.

5. Beiser, L. Proc. 1975 Electro-Opt. Syst. Des. Conf. 1975, 333.

6. Beiser, L.; Darcey, E.; Kleinschmitt, D. Proc. 1973 Electro-Opt. Syst. Des. Conf. 1973, 75. 
7. Beiser, L. Holographic Scanning; Wiley: New York, 1988.

8. Sincerbox, G.T. Laser Beam Scanning; Marshall, G., Ed.; Marcel Dekker: New York, 1985; 1.

9. Gabor, D. Nature 1948, 161, 777.

10. Leith, E.; Upatnieks, J. J. Opt. Soc. Am. 1962, 52, 1123.

11. Dickson, L.D.; Sincerbox, G.T.; Wolfheimer, A.D. IBM J. Res. Dev. 1982, 26, 228.

12. Pole, R.V.; Werlich, H.W.; Krusche, R. Appl. Opt. 1978, 17, 3294.

13. Dickson, L.D. Appl. Opt. 1970, 9, 1854.

14. Bartolini, R.A. Proc. SPIE 1977, 123, 2.

15. Smith, H.M., Ed. Holographie Recording Materials; Springer-Verlag: New York, 1977.

16. Werlich, H.; Sincerbox, G.; Yung, B. Dig. 1983 Conf. Lasers Electro-Opt. 1983, 224.

17. Werlich, H.; Sincerbox, G.; Yung, B. J. Imaging Tech. 1984, 10(3); 105.

18. Rogers, G. J. Opt. Soc. Amer. 1965, 55, 1185.

19. Upatnieks, J.; Leonard, C. Appl. Opt. 1969, 8, 85.

20. Pennington, K.; Harper, J. Appl. Opt. 1970, 9, 1643.

21. Graube, A. Appl. Opt. 1974, 13, 2942.

22. Phillips, N.; Porter, D. J. Phys. E. 1976, 9, 631.

23. Phillips, N.; Cullen, R.; Ward, A.; Porter, D. Photogr. Sei. Eng. 1980, 24, 120.

24. Booth, B. J. Appl. Phot. Eng. 1977, 3, 24.

25. Chandross, E.; Tomlinson, W.; Aumiller, G. Appl. Opt. 1978, 17, 566.

26. Kogelnik, H. Bell. Sys. Tech. J. 1969, 48, 2909.

27. Gambogi, W.J.; Gerstadt, W.A.; Mackara, S.R.; Weber, A.M. Proc. SPIE 1991, 1555, 256.

28. Ingwall, R. Proc. SPIE 1986, 615, 81.

29. Shankoff, T. Appl. Opt. 1968, 7, 2101.

30. Lin, L. Appl. Opt. 1969, 8, 903.

31. Chang, B.J. Opt. Eng. 1980, 19, 642.

32. Meyerhofer, D. RCA Rev. 1972, 33, 111.

33. Samoilovich, D.; Zeichner, A.; Freisem, A. Photogr. Sei. Eng. 1980, 24, 161.

34. Sjolinder, S. Photogr. Sei. Eng. 1981, 25, 112.

35. Chang, B.J.; Leonard, C.D. Appl. Opt. 1979, 18, 2407.

36. Graube, A. Opt. Commun. 1973, 8, 251.

37. Graube, A. Photogr. Sei. Eng. 1978, 22, 37.

38. Kubota, T.; Ose, T. Appl. Opt. 1979, 18, 2538.

39. Akagi, M. Photogr. Sei. Eng. 1974, 18, 248.

40. Kubota, T.; Ose, T.; Sasaki, M.; Honda, M. Appl. Opt. 1976, 15, 556.

41. Dickson, L.D. U.S. Patent 4,416,505, assigned to IBM, November 22, 1983.

42. Lerner, J.; Flamand, J.; Thevenon, A. Proc. SPIE 1982, 353, 68.

43. Ruda, J.C. J. Audio Eng. Soc. 1977, 25, 702.

44. Roys, W.E., Ed. Disc Recording and Reproduction; Dowden, Hutchinson \& Ross: Stroudsburg, PA, 1978.

45. Bartolini, R.; Feldstein, N.; Ryan, R.J. J. Electrochem. Soc. 1973, 120, 1408.

46. Gale, M.T.; Kane, J.; Knop, K. J. Appl. Phot. Eng. 1978, $4,41$.

47. Hanak, J.J.; Russell, J.P. RCA Rev. 1971, 32, 319.

48. Lehman, H.W.; Widner, R. J. Vac. Sci. Tech. 1980, 17, 1177.

49. Matsui, S.; Moriwaki, K.; Aritome, H.; Namba, S.; Shin, S.; Suga, S. Appl. Opt. 1982, 21, 2787.

50. Iwata, F.; Tsujiuchi, J. Appl. Opt. 1974, 13, 1327.

51. Wolpert, H.D. Photonics spectra 1983, 17(2-3), 68.

52. Ryan, R.J. RCA Rev. 1978, 39, 87.

53. Okino, Y.; Sano, K.; Kashihara, T. Proc. SPIE 1982, 329, 236. 